\title{
Phase-field systems with nonlinear coupling and dynamic boundary conditions
}

\author{
Cecilia Cavaterra \\ Dipartimento di Matematica "F. Enriques" \\ Università degli Studi di Milano, \\ 20133 Milano, ITALY \\ cecilia.cavaterra@unimi.it \\ Ciprian G. Gal \\ Department of Mathematics \\ University of Missouri, \\ Columbia, MO 65211, USA \\ ciprian@math.missouri.edu \\ Maurizio Grasselli \\ Dipartimento di Matematica "F. Brioschi" \\ Politecnico di Milano \\ 20133 Milano, ITALY \\ maurizio.grasselli@polimi.it \\ Alain Miranville \\ Université de Poitiers \\ Laboratoire de Mathématiques et Applications \\ UMR CNRS 6086, SP2MI \\ 86962 Chasseneuil Futuroscope Cedex, France \\ miranv@math.univ-poitiers.fr \\ March 16, 2009
}




\begin{abstract}
We consider phase-field systems of Caginalp type on a three-dimensional bounded domain. The order parameter $\psi$ fulfills a dynamic boundary condition, while the (relative) temperature $\theta$ is subject to a boundary condition of Dirichlet, Neumann or Robin type. Moreover, the two equations are nonlinearly coupled through a quadratic growth function. Here we extend a number of results which have been proven by some of the authors for the linear coupling. More precisely, we demonstrate the existence and uniqueness of global solutions. Then we analyze the associated dynamical system and we establish the existence of global as well as exponential attractors. We also discuss the convergence of given solutions to single equilibria.
\end{abstract}

\title{
1 Introduction
}

A well-known system of partial differential equations which describes the behavior of a twophase material in presence of temperature variations, and neglecting mechanical stresses, is the so-called Caginalp phase-field system (cf. [6, 11], see also [5, 38])

$$
\left\{\begin{array}{c}
\delta \psi_{t}-\Delta \psi+f(\psi)-\lambda^{\prime}(\psi) \theta=0, \\
(\varepsilon \theta+\lambda(\psi))_{t}-\Delta \theta=0,
\end{array}\right.
$$

in $\Omega \times(0,+\infty)$, where $\Omega \subset \mathbb{R}^{3}$ is a bounded domain occupied by the material for any time $t \geq 0$. Here $\psi$ is the order parameter (or phase-field), $\theta$ denotes the (relative) temperature, $\delta$ and $\varepsilon$ are given positive constants, while $\lambda$ is a function related to the latent heat. Moreover, the function $f: \mathbb{R} \rightarrow \mathbb{R}$ accounts for the presence of different phases (typically $f$ can be the derivative of a double well potential, i.e., $\left.f(y)=y^{2}(y-1)\right)$.

The mathematical literature regarding system (1.1) is rather vast when $\lambda$ is assumed to be linear. For instance, we recall that global well-posedness results can be found in [15] (see also $[12,44,45]$ ). The analysis of the longtime behavior of solutions to equations like (1.1) was also carried out in a number of papers mainly devoted to establish the existence of global and/or exponential attractors (see, for instance, [2, 3, 4, 30, 31, 32]). Moreover, the asymptotic behavior of single solutions has been investigated by means of suitable versions of the Łojasiewicz-Simon inequality (cf., e.g., [9, 27, 53]). All the mentioned results are essentially concerned with standard boundary conditions (that is, Dirichlet's, Neumann's or Robin's). More recently, in order to account for possible interactions with the walls, i.e., with the boundary $\Gamma:=\partial \Omega$, some physicists proposed a dynamic boundary condition for $\psi$ (cf., e.g., [16, 17, 33]). On account of this proposal, here we consider system (1.1) endowed with the following boundary conditions

$$
\left\{\begin{array}{c}
\psi_{t}-\alpha \Delta_{\Gamma} \psi+\partial_{\mathbf{n}} \psi+\beta \psi+g(\psi)=0 \\
b \partial_{\mathbf{n}} \theta+c \theta=0
\end{array}\right.
$$

\footnotetext{
${ }^{1} 2000$ Mathematics Subject Classification: 35B40, 35B41, 35K55, 80A22.

Keywords: phase-field equations, dynamic boundary conditions, Laplace-Beltrami operator, global attractors, exponential attractors, Łojasiewicz-Simon inequality, convergence to equilibrium.
} 
on $\Gamma \times(0,+\infty)$. Here $\alpha, \beta>0$ are given constants, $\mathbf{n}$ is the outward normal to $\Gamma, \Delta_{\Gamma}$ denotes the Laplace-Beltrami operator on $\Gamma$, and $g: \mathbb{R} \rightarrow \mathbb{R}$ is a given nonlinear function. $\Gamma$ is always supposed as smooth as is needed. The homogeneous boundary condition for $\theta$ subsumes the following cases: Dirichlet's $(b=0$ and $c>0)$, Neumann's $(b>0$ and $c=0)$, Robin's $(b>0$ and $c>0$ ).

Equations (1.1)-(1.2) are also subject to the initial conditions

$$
\psi_{\mid t=0}=\psi_{0}, \quad \theta_{\mid t=0}=\theta_{0}, \quad \text { in } \Omega
$$

Initial and boundary value problems (1.1)-(1.3), always in the case of a linear $\lambda$, were first analyzed in the case $(b, c)=(1,0)$. More precisely, well-posedness and convergence to single stationary states were demonstrated in [7] taking $g \equiv 0$ and assuming $f$ with a polynomially controlled growth of degree six. Existence of global and exponential attractors was proven in [24], under rather weak assumptions on $f$ and $g$ (see also [22]). These results were further generalized by assuming dynamic boundary conditions for $\theta$ in [20] and [21].

Assuming $\lambda$ linear is satisfactory for solid-liquid phase transitions. However, when one deals, for instance, with phase transitions in ferromagnetic materials, where $\psi$ represents the fraction of lattice sites at which the spins are pointing "up", then a quadratic $\lambda$ is a more appropriate choice (see [5] and references therein). In this paper, all the existing results (see $[28,34]$, cf. also [25], for memory effects, and [26, Sec. 7]) are concerned with homogeneous Neumann boundary condition for $\psi$. Here we want to extend the results contained in [20] and [21] to the case of a $\lambda$ with quadratic growth, under sufficiently general assumptions on $f$ and $g$, leaving the analysis of dynamic coupled boundary conditions (see [22]) and/or singular potentials (cf. $[8,10])$ to further investigations.

To be more precise, let us sketch the plan of the paper. In Section 2 we introduce the functional framework associated with (1.1)-(1.3). Then we state and prove the well-posedness results for our problems distinguishing between weak solutions (with growth restrictions on $f$ and $g$ ) and strong solutions (no growth restrictions on $f$ and $g$ ). In Section 3 we first demonstrate the existence of bounded absorbing sets and of compact absorbing sets. Hence, we deduce the existence of global attractors either for weak solutions and for strong ones. Section 4 is devoted to the existence of exponential attractors. Finally, in Section 5 we study the convergence of solutions to single equilibria.

\section{Well-posedness}

Without loss of generality, from now on we let $\delta=\varepsilon=1$. We denote by $\|\cdot\|_{p}$ and $\|\cdot\|_{p, \Gamma}$, the norms on $L^{p}(\Omega)$ and $L^{p}(\Gamma)$, respectively. In the case $p=2,\langle\cdot, \cdot\rangle_{2}\left(\right.$ or $\left.\langle\cdot, \cdot\rangle_{2, \Gamma}\right)$ stands for the usual scalar product which induces the $L^{2}$ norm (even for vector-valued functions). The norms on $H^{s}(\Omega)$ and $H^{s}(\Gamma)$ are indicated by $\|\cdot\|_{H^{s}(\Omega)}$ and $\|\cdot\|_{H^{s}(\Gamma)}$, respectively, for any $s>0$. In order to account for all the cases, we also introduce the family of linear operators 
$A_{K}:=-\Delta$ on the Banach space $L^{2}(\Omega)$ with domain

$$
\begin{aligned}
& D\left(A_{K}\right)=H_{0}^{1}(\Omega) \cap H^{2}(\Omega), \quad \text { if } K=D, \\
& D\left(A_{K}\right)=\left\{u \in H^{2}(\Omega): b \partial_{\mathbf{n}} \theta+c \theta=0, \quad \text { a.e. on } \Gamma\right\}, \quad \text { if } K \in\{N, R\},
\end{aligned}
$$

where $K \in\{D, N, R\}$ and $D, N, R$ stand for Dirichlet, Neumann, or Robin boundary conditions, respectively.

We recall that $A_{K}$ generates an analytic semigroup $e^{-A_{K} t}$ on $L^{2}(\Omega)$. In addition, each $A_{K}$ is nonnegative and self-adjoint on $L^{2}(\Omega)$. For our convenience, we also set

$$
\mathbb{Z}_{D}^{1}=H_{0}^{1}(\Omega), \quad \mathbb{Z}_{K}^{1}=H^{1}(\Omega), \text { if } K \in\{N, R\}
$$

endowed with the norms defined by

$$
\|\theta\|_{\mathbb{Z}_{K}^{1}}^{2}=\left\{\begin{array}{c}
\|\nabla \theta\|_{2}^{2}, \quad \text { if } K=D, \\
\|\nabla \theta\|_{2}^{2}+\frac{c}{b}\left\|\theta_{\mid \Gamma}\right\|_{2, \Gamma}^{2}, \quad \text { if } K=R, \\
\|\nabla \theta\|_{2}^{2}+\langle\theta\rangle_{\Omega}^{2}, \quad \text { if } K=N,
\end{array}\right.
$$

where we have set

$$
\langle v\rangle_{\Omega}:=|\Omega|^{-1} \int_{\Omega} v(x) d x .
$$

It is easy to check that, for each $K \in\{D, N, R\}$, the norm in $\mathbb{Z}_{K}^{1}$ is equivalent to the standard $H^{1}$-norm. On account of the dynamic boundary condition (see (1.2)), we also need to introduce the functional spaces $\mathbb{V}_{s}={\overline{C^{s}(\bar{\Omega})}}^{\|\cdot\|_{\mathbb{V}_{s}}}$, where $s>0$ and the norms $\|\cdot\|_{\mathbb{V}_{s}}$ are given by

$$
\|\psi\|_{\mathbb{V}_{s}}=\left(\|\psi\|_{H^{s}(\Omega)}^{2}+\left\|\psi_{\mid \Gamma}\right\|_{H^{s}(\Gamma)}^{2}\right)^{1 / 2} .
$$

It easy to see that $\mathbb{V}_{s}=H^{s}(\Omega) \oplus H^{s}(\Gamma)$. We also set $\mathbb{V}_{0}=L^{2}(\Omega) \oplus L^{2}(\Gamma)$. Furthermore, we notice that $\mathbb{V}_{s}$ is compactly contained in $\mathbb{V}_{s-1}$, for all $s \geq 1$. We also recall that we have the following continuous embeddings: $H^{1}(\Omega) \subset L^{6}(\Omega), H^{1 / 2}(\Gamma) \subset L^{4}(\Gamma)$ and $H^{1}(\Gamma) \subset L^{s}(\Gamma)$, for any fixed $s \in[1,+\infty)$. For the latter embedding, note that $\bar{\Omega}$ is a smooth, compact 3-dimensional Riemannian manifold whose boundary $\Gamma$ is a 2 -dimensional Riemannian manifold (see, e.g., [13, Chapter 2]).

Let $K \in\{D, N, R\}$. We now restate problem (1.1)-(1.3) in weak and strong formulations. In the case $K=N$ we define the enthalpy

$$
I_{N}(u, v):=\langle\lambda(u)+v\rangle_{\Omega},
$$

a quantity which is conserved in time.

Problem $\mathbf{P}_{K}^{w}$. For any given pair of initial data $\left(\psi_{0}, \theta_{0}\right) \in \mathbb{V}_{1} \times L^{2}(\Omega)$, find

$$
(\psi, \theta) \in C\left([0,+\infty) ; \mathbb{V}_{1} \times L^{2}(\Omega)\right),
$$


with

$$
\psi_{t} \in L^{2}\left([0,+\infty) ; \mathbb{V}_{0}\right), \quad \nabla \theta \in L^{2}\left([0,+\infty) ;\left(L^{2}(\Omega)\right)^{3}\right)
$$

such that

$$
\begin{aligned}
& \left\langle\psi_{t}, u\right\rangle_{2}+\langle\nabla \psi, \nabla u\rangle_{2}+\left\langle f(\psi)-\lambda^{\prime}(\psi) \theta, u\right\rangle_{2} \\
& +\left\langle\psi_{t}, u\right\rangle_{2, \Gamma}+\alpha\left\langle\nabla_{\Gamma} \psi, \nabla_{\Gamma} u\right\rangle_{2, \Gamma}+\langle\beta \psi+g(\psi), u\rangle_{2, \Gamma}=0, \quad \forall u \in \mathbb{V}_{1} \text {, a.e. in }(0, \infty), \\
& \left\langle(\theta+\lambda(\psi))_{t}, v\right\rangle_{2}+\langle\nabla \theta, \nabla v\rangle+d\langle\theta, v\rangle_{2, \Gamma}=0, \quad \forall v \in \mathbb{Z}_{K}^{1}, \text { a.e. in }(0, \infty),
\end{aligned}
$$

which satisfies (1.3) and, if $K=N$,

$$
I_{N}(\psi(t), \theta(t))=I_{N}\left(\psi_{0}, \theta_{0}\right), \quad \forall t \geq 0 .
$$

Here $d=\frac{c}{b}$ if $K=R, d=0$ otherwise.

Problem $\mathbf{P}_{K}^{s}$. For any given pair of initial data $\left(\psi_{0}, \theta_{0}\right) \in \mathbb{V}_{2} \times \mathbb{Z}_{K}^{1}$, the weak solution $(\psi, \theta)$ is such that

$$
(\psi, \theta) \in C\left([0,+\infty) ; \mathbb{V}_{2} \times \mathbb{Z}_{K}^{1}\right)
$$

with

$$
\left(\psi_{t}, \theta_{t}\right) \in L^{2}\left([0,+\infty) ; \mathbb{V}_{1} \times L^{2}(\Omega)\right),
$$

Our goal is to prove that both $\mathbf{P}_{K}^{w}$ and $\mathbf{P}_{K}^{s}$ have a unique global solution which continuously depends on the initial data. However, we first need to specify some assumptions on the nonlinearities $f, g$ and $\lambda$.

Here below we list some assumptions on $f, g, \lambda$ which will be used in the sequel.

(H1) $f, g \in C^{1}(\mathbb{R})$ satisfy

$$
\lim _{|y| \rightarrow+\infty} \inf f^{\prime}(y)>0, \quad \lim _{|y| \rightarrow+\infty} \inf g^{\prime}(y)>0 .
$$

(H2) $f$ and $g$ satisfy the growth assumptions

$$
\left|f^{\prime}(y)\right| \leq c_{f}\left(1+|y|^{2}\right), \quad\left|g^{\prime}(y)\right| \leq c_{g}\left(1+|y|^{q}\right), \quad \forall y \in \mathbb{R},
$$

for some positive constants $c_{f}, c_{g}$, where $q \in[1,+\infty)$ is arbitrary.

(H3) $\lambda \in C^{2}(\mathbb{R})$ satisfies

$$
\left|\lambda^{\prime \prime}(y)\right| \leq c_{\lambda}, \quad \forall y \in \mathbb{R}
$$

for some positive constant $c_{\lambda}$.

(H4) $\lambda$ has the form

$$
\lambda(y)=\gamma(y)-a y^{2}, \quad \forall y \in \mathbb{R},
$$

where $a>0$ and $\gamma \in C^{2}(\mathbb{R})$ such that $\gamma^{\prime} \in L^{\infty}(\mathbb{R})$.

(H5) $f$ satisfies

$$
f(y) y \geq \eta_{1}|y|^{4}-\eta_{2}, \quad \forall y \in \mathbb{R},
$$

for some constants $\eta_{1}>0$ and $\eta_{2} \geq 0$. 
Remark 2.1 Assumption (H4) is needed only to handle the case $K=N$, otherwise (H5) suffices. Note that (H4) is justified from a physical viewpoint (see, e.g., [5, Chap. 4]).

We now state and prove the first global existence result.

Theorem 2.2 Let $f, g, \lambda$ satisfy assumptions (H1)-(H3). Then, for each $K \in\{D, N, R\}$, problem $\mathbf{P}_{\mathbf{K}}^{w}$ admits a global weak solution.

Proof. A unique local (and sufficiently smooth) solution to problem $\mathbf{P}_{\mathbf{K}}^{w}$ can be found by means of a suitable application of the Faedo-Galerkin approximation scheme (see [18], for a similar problem). To apply such an argument to a problem with a dynamic boundary condition, we need to construct suitable self-adjoint operators acting on $\mathbb{V}_{0}$. Such constructions have already appeared in [19]. To this end, let us consider the operator $\mathcal{B}_{0}$ given formally by

$$
\mathcal{B}_{0}\left(\psi, \psi_{\mid \Gamma}\right)=\left(-\Delta \psi,(-\Delta \psi)_{\mid \Gamma}\right),
$$

for functions $\psi \in C^{2}(\bar{\Omega})$, with $\psi_{\mid \Gamma} \in C^{2}(\Gamma)$, that satisfy the Wentzell boundary condition

$$
\Delta \psi-\alpha \Delta_{\Gamma} \psi_{\mid \Gamma}+\partial_{\mathbf{n}} \psi+\beta \psi_{\mid \Gamma}=0 \text { on } \Gamma .
$$

Here $(\Delta \psi)_{\mid \Gamma}$ stands for the restriction of the Laplace operator $\Delta$ on the boundary $\Gamma$ and it should not be confused with the Laplace-Beltrami operator $\Delta_{\Gamma} \psi_{\mid \Gamma}$. The domain of $\mathcal{B}_{0}$ is $D\left(\mathcal{B}_{0}\right)=\left\{\Theta=\left(\psi, \psi_{\mid \Gamma}\right): \psi \in C^{2}(\bar{\Omega}),(2.9)\right.$ hold $\}$. It is an easy exercise (cf. [19]) to show that we have $\left\langle\mathcal{B}_{0} \Theta, \Xi\right\rangle_{\mathbb{V}_{0}}=\langle\Theta, \Xi\rangle_{\mathbb{V}_{1}}$, for all $\Theta \in D\left(\mathcal{B}_{0}\right)$ and $\Xi \in \mathbb{V}_{1}$. It follows that $\mathcal{B}_{0}$ is symmetric on $\mathbb{V}_{0}$. Let us now consider a function $f \in C(\bar{\Omega})$ such that $F=\left(f_{1}, f_{2}\right)$ with $f_{1}:=\left.f\right|_{\Omega}$ and $f_{2}:=\left.f\right|_{\Gamma}$. By the equality $\mathcal{B}_{0} \Theta=F$, we mean the following boundary value problem:

$$
\begin{array}{lll}
-\Delta \psi=f_{1} & \text { in } \quad & \Omega, \\
-\Delta \psi=f_{2} & \text { on } \quad \Gamma .
\end{array}
$$

Using the Wentzell boundary condition (2.9) and replacing $f_{2}$ by $f_{\mid \Gamma}$, the boundary condition (2.11) becomes

$$
-\alpha \Delta_{\Gamma} \psi_{\mid \Gamma}+\partial_{\mathbf{n}} \psi+\beta \psi_{\mid \Gamma}=f_{2} \text { on } \Gamma .
$$

We now define the "Wentzell version of $\mathcal{B}_{0}$ ", $\widetilde{\mathcal{B}}_{0}$, by $\mathcal{B}_{0} \Theta=F$ on

$$
\begin{aligned}
D\left(\widetilde{\mathcal{B}}_{0}\right) & =\left\{\Theta \in \mathbb{V}_{0}: \Theta \text { corresponds to } \psi \in H^{2}(\Omega), \psi_{\mid \Gamma} \in H^{2}(\Gamma),\right. \\
& \text { and }(2.10),(2.12) \text { hold }\} .
\end{aligned}
$$

Let $\mathcal{B}$ be the closure of $\widetilde{\mathcal{B}}_{0}$. Then, using the techniques in [19], we can check that $\mathcal{B}$ is selfadjoint and positive on $\mathbb{V}_{0}$, since $\beta>0 ; \mathcal{B}$ is the operator associated with the positive symmetric closed bilinear form $\langle\Theta, \Xi\rangle_{\mathbb{V}_{1}}$. Also by [19], the following characterization of the domain of $\mathcal{B}$ is given, provided that $\Gamma$ is smooth enough:

$$
D(\mathcal{B})=\left\{\psi \in H^{2}(\Omega): \psi_{\mid \Gamma} \in H^{2}(\Gamma)\right\} .
$$


Thus, for $i \in \mathbf{N}$, we take a complete system of eigenfunctions $\left\{\Theta_{i}\right\}$ of the problem $\mathcal{B} \Theta_{i}=$ $\widehat{\lambda}_{i} \Theta_{i}$ in $\mathbb{V}_{0}$ with $\Theta_{i} \in D(\mathcal{B})$. Moreover, recall that $A_{K}=-\Delta$, when $K \in\{D, N, R\}$ is nonnegative and self-adjoint operator on $L^{2}(\Omega)$. Then, we have (for $i \in \mathbf{N}$ ) a complete system of eigenfunctions $\left\{\xi_{i}^{K}\right\}$ of the problem $A_{K} \xi_{i}^{K}=\lambda_{i}^{K} \xi_{i}^{K}$ in $L^{2}(\Omega)$ with $\xi_{i}^{K} \in D\left(A_{K}\right)$. According to the general spectral theory, the eigenvalues $\widehat{\lambda}_{i}$ and $\lambda_{i}^{K}, K \in\{D, N, R\}$ can be increasingly ordered and counted according to their multiplicities in order to form a real divergent sequence. Moreover, the respective eigenvectors $\Theta_{i}$ and $\xi_{i}^{K}$ turn out to form an orthogonal basis in $\mathbb{V}_{1}, \mathbb{V}_{0}$ and $L^{2}(\Omega), \mathbb{Z}_{K}^{1}$ respectively. The eigenvectors $\Theta_{i}$ and $\theta_{i}^{K}$ may be assumed to be normalized in the norm of $\mathbb{V}_{0}$ and $L^{2}(\Omega)$ respectively. At this point, we set the spaces

$$
\begin{gathered}
\mathcal{K}_{n}=\operatorname{span}\left\{\Theta_{1}, \Theta_{2}, \ldots, \Theta_{n}\right\}, \mathcal{K}_{\infty}=\cup_{n=1}^{\infty} \mathcal{K}_{n}, \\
\mathcal{P}_{n}^{K}=\operatorname{span}\left\{\xi_{1}^{K}, \xi_{2}^{K}, \ldots, \xi_{n}^{K}\right\}, \mathcal{P}_{\infty}^{K}=\cup_{n=1}^{\infty} \mathcal{P}_{n}^{K} .
\end{gathered}
$$

Clearly, $\mathcal{K}_{\infty}$ and $\mathcal{P}_{\infty}^{K}, K \in\{D, N, R$,$\} are dense subspaces of \mathbb{V}_{1}, \mathbb{V}_{2}$ and $\mathbb{Z}_{K}^{1}, D\left(A_{K}\right)$ respectively. For any $n \in \mathbf{N}$, we look for functions of the form

$$
\psi=\psi_{n}=\sum_{i=1}^{n} d_{i}(t) \Theta_{i}, \theta^{K}=\theta_{n}^{K}=\sum_{i=1}^{n} e_{i}(t) \xi_{i}^{K}
$$

solving the approximate problem that we will introduce below. In the definition of $\psi_{n}$ and $\theta_{n}^{K}, d_{i}(t)$ and $e_{i}(t)$ are sought to be suitably regular real valued functions. As approximations for the initial data $\left(\psi_{0}, \theta_{0}\right)$, we take $\left(\psi_{n 0}, \theta_{n 0}\right) \in \mathbb{V}_{1} \times L^{2}(\Omega)$, such that

$$
\lim _{n \rightarrow \infty}\left(\psi_{n 0}, \theta_{n 0}\right)=\left(\psi_{0}, \theta_{0}\right) \text { in } \mathbb{V}_{1} \times L^{2}(\Omega) .
$$

The problem that we must solve is given by $\mathbf{P}_{\mathbf{K}}^{w, n}$, for a fixed $K \in\{D, N, R\}$ and for any $n \geq 1$,

$$
\left\{\begin{array}{c}
\left\langle\partial_{t} \psi_{n}, \bar{\Theta}\right\rangle_{\mathbb{V}_{0}}+\left\langle\mathcal{B} \psi_{n}, \bar{\Theta}\right\rangle_{\mathbb{V}_{0}}+\left\langle f\left(\psi_{n}\right), \bar{\Theta}\right\rangle_{2}+\left\langle g\left(\psi_{n}\right), \bar{\Theta}\right\rangle_{2, \Gamma}=-\left\langle\lambda^{\prime}\left(\psi_{n}\right) \theta_{n}^{K}, \bar{\Theta}\right\rangle_{2}, \\
\left\langle\partial_{t} \theta_{n}^{K}, \bar{\theta}\right\rangle_{2}+\left\langle A_{K} \theta_{n}^{K}, \bar{\theta}\right\rangle_{2}=-\left\langle\lambda^{\prime}\left(\psi_{n}\right) \partial_{t} \psi_{n}, \bar{\theta}\right\rangle_{2},
\end{array}\right.
$$

and

$$
\left\langle\psi_{n}(0), \bar{\Theta}\right\rangle_{\mathbb{V}_{0}}=\left\langle\psi_{n 0}, \bar{\Theta}\right\rangle_{\mathbb{V}_{0}},\left\langle\theta_{n}^{K}(0), \bar{\theta}\right\rangle_{2}=\left\langle\theta_{n 0}, \bar{\theta}\right\rangle_{2},
$$

for all $\bar{\Theta} \in \mathcal{K}_{n}, \bar{\theta} \in \mathcal{P}_{n}^{K}$.

We aim to apply the standard existence theorems for ODE's. For this purpose, if $n$ is fixed, let us choose $\bar{\Theta}=\Theta_{j}$, and $\bar{\theta}=\xi_{j}^{K}, 1 \leq j \leq n$ and substitute the expressions (2.13) to the unknowns $\psi_{n}$ and $\theta_{n}^{K}$ in (2.15)-(2.16). After performing direct computations (and using the above definitions), we can transform our problem and the initial conditions of $\mathbf{P}_{\mathbf{K}}^{w, n}$ into a Cauchy problem for a system of nonlinear ordinary differential equations:

$$
\left\{\left(\begin{array}{c}
\partial_{t} \mathbf{e}(t) \\
\partial_{t} \mathbf{d}(t)
\end{array}\right)=\left(\begin{array}{c}
\mathbb{U}_{1}(t, \mathbf{d}(t), \mathbf{e}(t)) \\
\mathbb{U}_{2}(t, \mathbf{d}(t), \mathbf{e}(t))
\end{array}\right)\right.
$$

where $\mathbb{U}_{l}:\left[0, t_{n}\right] \times \mathbb{R}^{2 n} \rightarrow \mathbb{R}^{2 n}, l=1,2$, can be computed explicitly. Each function $\mathbb{U}_{l}$, $l=1,2$ is continuous with respect to its arguments, uniformly in $t$, by the continuity of 
the projections and the fact that $f, g$ and $\lambda$ are continuous. Applying Cauchy's theorem for ODE's, we find a small time $t_{n} \in(0, T)$ such that (2.15)-(2.16) holds for all $t \in\left[0, t_{n}\right]$. This gives the desired local $C^{1}$-solution $\left(\psi_{n}, \theta_{n}^{K}\right)$ to our problem. We will now deduce a priori estimates for the solutions $\left(\psi_{n}, \theta_{n}^{K}\right)$. Such estimates, in particular, ensure that we can take $t_{n}=T$, for every $n$. Throughout this proof, $C$ will denote a positive generic constant, depending at most on the physical parameters of the problem, but independent of $n$. This constant may vary even in the same line. Further dependence of the constants will be pointed out if needed.

Let us fix $K \in\{D, N, R\}$ and consider our local (sufficiently smooth) continuous solutions $\left(\psi_{n}, \theta_{n}^{K}\right)$. For the sake of exposition, we will drop the superscript $K$ from $\theta_{n}^{K}$. We take $\bar{\theta}=\partial_{t} \psi_{n}(t)$ and $\bar{\Theta}=\theta_{n}(t)$ in (2.15). We thus obtain

$$
\begin{gathered}
\left\|\partial_{t} \psi_{n}(t)\right\|_{\mathbb{V}_{0}}^{2}+\frac{1}{2} \frac{d}{d t}\left[\alpha\left\|\nabla_{\Gamma} \psi_{n}(t)\right\|_{2, \Gamma}^{2}+\beta\left\|\psi_{n}(t)\right\|_{2, \Gamma}^{2}+\left\|\nabla \psi_{n}(t)\right\|_{2}^{2}\right] \\
+\int_{\Omega} f\left(\psi_{n}(t)\right) \partial_{t} \psi_{n}(t) d x+\int_{\Gamma} g\left(\psi_{n}(t)\right) \partial_{t} \psi_{n}(t) d S \\
=\left\langle\partial_{t} \psi_{n}(t), \lambda^{\prime}\left(\psi_{n}(t)\right) \theta_{n}(t)\right\rangle_{2}, \\
\frac{1}{2} \frac{d}{d t}\left\|\theta_{n}(t)\right\|_{2}^{2}+\left\|\nabla \theta_{n}(t)\right\|_{2}^{2}+d\left\|\theta_{n \mid \Gamma}(t)\right\|_{2, \Gamma}^{2}=-\left\langle\lambda^{\prime}\left(\psi_{n}(t)\right) \partial_{t} \psi_{n}(t), \theta_{n}(t)\right\rangle_{2} .
\end{gathered}
$$

Adding together the above equations, then integrating with respect to time and exploiting (2.14), we get

$$
\begin{aligned}
& \left\|\psi_{n}(t)\right\|_{\mathbb{V}_{1}}^{2}+\int_{0}^{t}\left\|\partial_{t} \psi_{n}(s)\right\|_{\mathbb{V}_{0}}^{2} d s+\left\|\theta_{n}(t)\right\|_{2}^{2} \\
& +\int_{0}^{t}\left(\left\|\nabla \theta_{n}(s)\right\|_{2}^{2}+d\left\|\theta_{n \mid \Gamma}(s)\right\|_{2, \Gamma}^{2}\right) d s+\int_{\Omega} F\left(\psi_{n}(t)\right) d x+\int_{\Gamma} G\left(\psi_{n}(t)\right) d S \\
& \leq C
\end{aligned}
$$

where

$$
F(y)=\int_{0}^{y} f(r) d r, \quad G(y)=\int_{0}^{y} g(r) d r .
$$

Recalling that $F$ and $G$ are bounded from below (independently of $n$ ) due to (2.5), we infer

$$
\left\|\psi_{n}(t)\right\|_{\mathbb{V}_{1}}^{2}+\int_{0}^{t}\left\|\partial_{t} \psi_{n}(s)\right\|_{\mathbb{V}_{0}}^{2} d s+\left\|\theta_{n}(t)\right\|_{2}^{2}+\int_{0}^{t}\left(\left\|\nabla \theta_{n}(s)\right\|_{2}^{2}+d\left\|\theta_{n \mid \Gamma}(s)\right\|_{2, \Gamma}^{2}\right) d s \leq C,
$$


for all $t \geq 0$. Observe that in the case $K=N$ (i.e., $d=0$ ), we need also to control $\left\langle\theta_{n}(t)\right\rangle_{\Omega}^{2}$. Hence, recalling (2.4), (2.7) and (2.20), we have

$$
|\Omega|^{-2}\left(\int_{\Omega} \theta_{n}(x, t) d x\right)^{2} \leq I_{N}^{2}\left(\psi_{0}, \theta_{0}\right)+C\left(1+\left\|\psi_{n}(t)\right\|_{2}^{4}\right) \leq C .
$$

In particular, the above bounds imply that the local solution $\left(\psi_{n}, \theta_{n}\right)$ to $\mathbf{P}_{\mathbf{K}}^{w, n}$ can be extended up to time $T$, that is, $t_{n}=T$, for every $n$.

From (2.20)-(2.21), we also learn that the sequence $\left(\psi_{n}, \theta_{n}\right)$ is uniformly bounded in norm in the space $\mathbb{V}_{1} \times L^{2}(\Omega)$ by a constant depending only on the size of initial data. In detail, for each fixed $T>0$, we have

$$
\begin{gathered}
\left\|\psi_{n}\right\|_{L^{\infty}\left([0, T] ; \mathbb{V}_{1}\right) \cap H^{1}\left([0, T] ; \mathbb{V}_{0}\right)} \leq C, \\
\left\|\theta_{n}\right\|_{L^{\infty}\left([0, T] ; L^{2}(\Omega)\right) \cap L^{2}\left([0, T] ; \mathbb{Z}_{K}^{1}\right)} \leq C .
\end{gathered}
$$

Exploiting the uniform bound of (2.22), we can also show

$$
\begin{aligned}
& \left\|\lambda^{\prime}\left(\psi_{n}\right) \partial_{t} \psi_{n}\right\|_{L^{2}\left([0, T] ;\left(\mathbb{Z}_{K}^{1}\right)^{*}\right)} \\
& \leq c_{\lambda}\left\|\partial_{t} \psi_{n}\right\|_{L^{2}\left([0, T] ; L^{2}(\Omega)\right)}\left(T^{1 / 4}+\left\|\psi_{n}\right\|_{L^{2}\left([0, T] ; H^{1}(\Omega)\right)}\right) \leq C .
\end{aligned}
$$

Finally, exploiting the uniform bounds (2.23)-(2.25) once again, from the second equation of (2.15), we deduce that

$$
\left\|\theta_{n}\right\|_{H^{1}\left([0, T] ;\left(\mathbb{Z}_{K}^{1}\right)^{*}\right)} \leq C
$$

We are ready to pass to the limit as $n$ goes to $+\infty$. On account of the above uniform inequalities, we can argue that, up to subsequences,

$$
\begin{aligned}
& \psi_{n} \rightarrow \psi \text { weakly }^{*} \text { in } L^{\infty}\left([0, T] ; \mathbb{V}_{1}\right), \\
& \partial_{t} \psi_{n} \rightarrow \partial_{t} \psi \text { weakly in } L^{2}\left([0, T] ; \mathbb{V}_{0}\right) \text {, }
\end{aligned}
$$

and

$$
\begin{aligned}
\theta_{n} & \rightarrow \theta \text { weakly* in } L^{\infty}\left([0, T] ; L^{2}(\Omega)\right), \\
\theta_{n} & \rightarrow \theta \text { weakly in } L^{2}\left([0, T] ; \mathbb{Z}_{K}^{1}\right), \\
\partial_{t} \theta_{n} & \rightarrow \partial_{t} \theta \text { weakly* in } L^{2}\left([0, T] ;\left(\mathbb{Z}_{K}^{1}\right)^{*}\right) .
\end{aligned}
$$

Due to (2.27)-(2.28) and classical compactness theorems, we also have

$$
\begin{aligned}
\psi_{n} & \rightarrow \psi \text { strongly in } C\left([0, T] ; \mathbb{V}_{1-s}\right), \\
\theta_{n} & \rightarrow \theta \text { strongly in } L^{2}\left([0, T] ; \mathbb{Z}_{K}^{1-s}\right),
\end{aligned}
$$

for all $s \in(0,1]$. By refining, $\psi_{n}$ converges to $\psi$ a.e. in $\Omega$ (pointwise in $[0, T]$ ) and $\theta_{n}$ converges to $\theta$ a.e. in $\Omega \times[0, T]$, respectively. Having now the uniform bounds $(2.22),(2.23)$ 
for the solutions $\left(\psi_{n}, \theta_{n}\right)$, we can easily control the nonlinear terms in the equations of (2.15). Since $\psi \in L^{\infty}\left([0, T] ; \mathbb{V}_{1}\right)$, it is easy to see, on the basis of assumption (H2), that

$$
\left|f\left(\psi_{n}\right)\right|_{L^{6}(\Omega \times[0, T])} \leq C, \quad\left|g\left(\psi_{n}\right)\right|_{L^{q}(\Gamma \times[0, T])} \leq C .
$$

Then, by means of known results of measure theory, the continuity of $f, g$ and (2.29), (2.30), we easily deduce that $f\left(\psi_{n}\right)$ converges weakly to $f(\psi)$ in $L^{6}(\Omega \times[0, T])$, and thus, weakly star in $L^{2}\left([0, T] ;\left(H^{1}(\Omega)\right)^{*}\right)$. Moreover, $g\left(\psi_{n}\right)$ converges weakly to $g(\psi)$ in $L^{q}(\Gamma \times[0, T])$, and thus, weakly star in $L^{q}\left([0, T] ;\left(H^{1}(\Gamma)\right)^{*}\right)$. On the other hand, on account of $(2.29)$ and (2.27)-(2.28), it is not difficult to show

$$
\lambda^{\prime}\left(\psi_{n}\right) \theta_{n} \rightarrow \lambda^{\prime}(\psi) \theta \text { weakly* in } L^{2}\left([0, T] ;\left(H^{1}(\Omega)\right)^{*}\right)
$$

and

$$
\lambda^{\prime}\left(\psi_{n}\right) \partial_{t} \psi_{n} \rightarrow \lambda^{\prime}(\psi) \partial_{t} \psi \text { weakly* in } L^{2}\left([0, T] ;\left(\mathbb{Z}_{K}^{1}\right)^{*}\right) .
$$

By means of the above convergences properties (2.27)-(2.32), we can pass to the limit in (2.15) to get all the equations of (2.2)-(2.3).

Finally, it is left to show that $\psi(0)=\psi_{0}$ and $\theta(0)=\theta_{0}$. But this follows from standard arguments and the above convergence properties of the solutions $\left(\psi_{n}, \theta_{n}\right)$. We omit the details. The proof of the theorem is finished.

We now state and prove the existence of a global strong solution.

Theorem 2.3 Let $f, g, \lambda$ satisfy (H1) and (H3). Then, for each $K \in\{D, N, R\}$, problem $\mathbf{P}_{\mathbf{K}}^{s}$ admits a global strong solution that satisfies

$$
\theta \in L^{2}\left([0,+\infty) ; D\left(A_{K}\right)\right)
$$

Proof. In Theorem 2.2, we have proved the existence of a sufficiently smooth global solution $(\psi, \theta)$ that was obtained as a limit (in appropriate topologies) of the solutions $\left(\psi_{n}, \theta_{n}\right)$ to problem (2.15)-(2.16). We need to obtain higher order estimates. Note first that equations (2.2)-(2.3) can be written now in the strong form and boundary conditions (1.2) hold almost everywhere in $\Gamma \times(0, T)$. Then we differentiate the first equation of $(2.15)$ with respect to time. Moreover, we multiply the resulting equation by $\partial_{t} \psi_{n}(t)$, and integrate over $\Omega$ and $\Gamma$, respectively. In addition, we multiply the second equation of $(2.15)$ by $\partial_{t} \theta_{n}(t)$ and integrate over $\Omega$ as well. Adding all the obtained relations and using Green's formula, we have

$$
\begin{aligned}
& \frac{1}{2} \frac{d}{d t}\left(\left\|\partial_{t} \psi_{n}(t)\right\|_{2}^{2}+\left\|\partial_{t} \psi_{n}(t)\right\|_{2, \Gamma}^{2}+\left\|\nabla \theta_{n}(t)\right\|_{2}^{2}+d\left\|\theta_{n \mid \Gamma}(t)\right\|_{2, \Gamma}^{2}\right) \\
& +\left\|\partial_{t} \psi_{n}(t)\right\|_{\mathbb{V}_{1}}^{2}+\left\|\partial_{t} \theta_{n}(t)\right\|_{2}^{2}+\int_{\Omega} f^{\prime}\left(\psi_{n}(t)\right)\left(\partial_{t} \psi_{n}(t)\right)^{2} d x+\int_{\Gamma} g^{\prime}\left(\psi_{n}(t)\right)\left(\partial_{t} \psi_{n}(t)\right)^{2} d S \\
& =\left\langle\lambda^{\prime \prime}\left(\psi_{n}(t)\right)\left(\partial_{t} \psi_{n}(t)\right)^{2}, \theta_{n}(t)\right\rangle_{2} .
\end{aligned}
$$

Thanks to (2.5), there holds

$$
-\int_{\Omega} f^{\prime}\left(\psi_{n}(t)\right)\left(\partial_{t} \psi_{n}(t)\right)^{2} d x-\int_{\Gamma} g^{\prime}\left(\psi_{n}(t)\right)\left(\partial_{t} \psi_{n}(t)\right)^{2} d S \leq C\left\|\partial_{t} \psi_{n}(t)\right\|_{\mathbb{V}_{0}}^{2}
$$


Moreover, using the interpolation inequality $\|u\|_{4} \leq c_{\Omega}\|u\|_{2}^{1 / 4}\|u\|_{H^{1}(\Omega)}^{3 / 4}$, then Young's inequality with exponents $(4 / 3,4)$, we estimate the term on the right-hand side of $(2.33)$, as follows (cf. (2.7))

$$
\begin{aligned}
\left|\left\langle\lambda^{\prime \prime}\left(\psi_{n}\right)\left(\partial_{t} \psi\right)^{2}, \theta_{n}\right\rangle_{2}\right| & \leq C\left\|\partial_{t} \psi_{n}\right\|_{4}^{2}\left\|\theta_{n}\right\|_{2} \\
& \leq C\left\|\partial_{t} \psi_{n}\right\|_{2}^{1 / 2}\left\|\partial_{t} \psi_{n}\right\|_{H^{1}(\Omega)}^{3 / 2}\left\|\theta_{n}\right\|_{2} \\
& \leq \eta\left(\left\|\nabla \partial_{t} \psi_{n}\right\|_{2}^{2}+\left\|\partial_{t} \psi_{n}\right\|_{2}^{2}\right)+C_{\eta}\left\|\partial_{t} \psi_{n}\right\|_{2}^{2}\left\|\theta_{n}\right\|_{2}^{4},
\end{aligned}
$$

for a sufficiently small $\eta \in(0,1)$ and large positive constant $C_{\eta}$. Then, on account of $(2.20)$ (2.35), an integration of (2.33) with respect to time yields

$$
\left\|\partial_{t} \psi_{n}(t)\right\|_{\mathbb{V}_{0}}^{2}+\left\|\nabla \theta_{n}(t)\right\|_{2}^{2}+d\left\|\theta_{n \mid \Gamma}(t)\right\|_{2, \Gamma}^{2}+\int_{0}^{t}\left(\left\|\partial_{t} \psi_{n}(s)\right\|_{\mathbb{V}_{1}}^{2}+\left\|\partial_{t} \theta_{n}(s)\right\|_{2}^{2}\right) d s \leq C
$$

for all $t \geq 0$. Note that, owing to (2.36), we can use the second equation of (2.2) to deduce

$$
\sup _{t \geq 0} \int_{0}^{t}\left\|\Delta \theta_{n}(s)\right\|_{2}^{2} d s \leq C
$$

To prove the last bound on $\psi_{n}$, we first need to apply the maximum principle to the following system (see [39, Lemma A.2])

$$
\left\{\begin{array}{c}
-\Delta \psi_{n}+f\left(\psi_{n}\right)=h_{1}:=-\partial_{t} \psi_{n}+\lambda^{\prime}\left(\psi_{n}\right) \theta_{n}, \quad \text { in } \Omega \times(0, T), \\
-\alpha \Delta_{\Gamma} \psi_{n}+\partial_{\mathbf{n}} \psi_{n}+\beta \psi_{n}+g\left(\psi_{n}\right)=h_{2}:=-\partial_{t} \psi_{n}, \quad \text { on } \Gamma \times(0, T),
\end{array}\right.
$$

which yields

$$
\left\|\psi_{n}(t)\right\|_{\infty}^{2}+\left\|\psi_{n}(t)\right\|_{\infty, \Gamma}^{2} \leq C\left(1+\left\|h_{1}(t)\right\|_{2}^{2}+\left\|h_{2}(t)\right\|_{2, \Gamma}^{2}\right)
$$

and, owing to (2.7), (2.20) and (2.36), it follows that

$$
\left\|\psi_{n}(t)\right\|_{\infty}^{2}+\left\|\psi_{n}(t)\right\|_{\infty, \Gamma}^{2} \leq C .
$$

It is worth recalling that [39, Lemma A.2] applies thanks to (2.5). On the other hand, using an $H^{2}$-regularity estimate (see, e.g., [39, Lemma A.1]), we have

$$
\left\|\psi_{n}(t)\right\|_{\mathbb{V}_{2}} \leq C\left(\left\|j_{1}(t)\right\|_{2}+\left\|j_{2}(t)\right\|_{2, \Gamma}\right)
$$

where

$$
j_{1}:=-\partial_{t} \psi_{n}-f\left(\psi_{n}\right)+\lambda^{\prime}\left(\psi_{n}\right) \theta_{n}, \quad j_{2}:=-\partial_{t} \psi_{n}-g\left(\psi_{n}\right) .
$$

Thus, thanks to (2.20), (2.36) and (2.40), from (2.41) we infer

$$
\left\|\psi_{n}(t)\right\|_{\mathbb{V}_{2}} \leq C
$$


Finally, observe that in the case $K=N$ we need to control also $\left\langle\theta_{n}(t)\right\rangle_{\Omega}^{2}$. But this can be done exactly as in (2.21).

It is easy to see that the local solution $\left(\psi_{n}, \theta_{n}\right)$ to problem $\mathbf{P}_{\mathbf{K}}^{s}$ is indeed global. Moreover, on account of (2.20), (2.36), (2.40), (2.43) and (2.21), we can argue again that, up to subsequences,

$$
\begin{aligned}
\psi_{n} & \rightarrow \psi \text { weakly }^{*} \text { in } L^{\infty}\left([0, T] ; \mathbb{V}_{2}\right), \\
\partial_{t} \psi_{n} & \rightarrow \partial_{t} \psi \text { weakly in } L^{2}\left([0, T] ; \mathbb{V}_{1}\right),
\end{aligned}
$$

and

$$
\begin{aligned}
\theta_{n} & \rightarrow \theta \text { weakly }^{*} \text { in } L^{\infty}\left([0, T] ; \mathbb{Z}_{K}^{1}\right), \\
\theta_{n} & \rightarrow \theta \text { weakly in } L^{2}\left([0, T] ; D\left(A_{K}\right)\right), \\
\partial_{t} \theta_{n} & \rightarrow \partial_{t} \theta \text { weakly in } L^{2}\left([0, T] ; L^{2}(\Omega)\right) .
\end{aligned}
$$

On the other hand, due to (2.44)-(2.45) and classical compactness theorems, we also have

$$
\begin{gathered}
\psi_{n} \rightarrow \psi \text { strongly in } C\left([0, T] ; \mathbb{V}_{2-s}\right), \\
\theta_{n} \rightarrow \theta \text { strongly in } C\left([0, T] ; \mathbb{Z}_{K}^{1-s}\right),
\end{gathered}
$$

for all $s \in(0,1]$. Furthermore, on account of (2.46) and the fact that $f$ and $g$ are continuously differentiable, it is not difficult to show

$$
\begin{gathered}
f\left(\psi_{n}\right) \rightarrow f(\psi) \text { strongly in } C\left([0, T] ; L^{2}(\Omega)\right), \\
g\left(\psi_{n}\right) \rightarrow g(\psi) \text { strongly in } C\left([0, T] ; L^{2}(\Gamma)\right) .
\end{gathered}
$$

Finally, each of the weak convergences of (2.32) and (2.44) are strong in the sense of $L^{2}\left([0, T] ; L^{2}(\Omega)\right)$. We can now pass to the limit to deduce the equations $(2.2)-(2.3)$ in the strong form. Note that the boundary conditions $(1.2)$ for $(\psi, \theta)$ hold almost everywhere in $\Gamma \times(0, T)$. We have finished the proof of the theorem.

Uniqueness of solutions to both problems $\mathbf{P}_{\mathbf{K}}^{w}$ and $\mathbf{P}_{\mathbf{K}}^{s}$ follows from the following continuous dependence estimates.

Lemma 2.4 Let the assumptions of Theorem 2.2 hold. Consider two global solutions $\left(\psi_{w i}, \theta_{w i}\right)$ to $\mathbf{P}_{\mathbf{K}}^{w}$ corresponding to the initial data $\left(\psi_{0 i}, \theta_{0 i}\right) \in \mathbb{V}_{1} \times L^{2}(\Omega), i=1,2$. Then, for any $t \geq 0$, the following estimate holds

$$
\begin{gathered}
\left\|\left(\psi_{w 1}-\psi_{w 2}\right)(t)\right\|_{\mathbb{V}_{1}}^{2}+\left\|\left(\theta_{w 1}-\theta_{w 2}\right)(t)\right\|_{2}^{2} \\
+\int_{0}^{t}\left[\left\|\left(\psi_{w 1}-\psi_{w 2}\right)_{t}(s)\right\|_{\mathbb{V}_{0}}^{2}+\left\|\left(\theta_{w 1}-\theta_{w 2}\right)(s)\right\|_{\mathbb{Z}_{K}^{1}}^{2}\right] d s \\
\leq C_{w} e^{L_{w} t}\left(\left\|\psi_{01}-\psi_{02}\right\|_{\mathbb{V}_{1}}^{2}+\left\|\theta_{01}-\theta_{02}\right\|_{2}^{2}\right),
\end{gathered}
$$

where $C_{w}$ and $L_{w}$ are positive constants depending on the norms of the initial data in $\mathbb{V}_{1} \times$ $L^{2}(\Omega)$, on $\Omega$ and on the parameters of the problem, but they are both independent of time. 
Proof. Let us set $\psi:=\psi_{1}-\psi_{2}$ and $\theta:=\theta_{1}-\theta_{2}$. Then we easily realize that $(\psi, \theta)$ solves the system

$$
\begin{aligned}
& \left\langle\psi_{t}, u\right\rangle_{2}+\langle\nabla \psi, \nabla u\rangle_{2}+\left\langle\Upsilon_{1}, u\right\rangle_{2}+\left\langle\psi_{t}, u\right\rangle_{2, \Gamma} \\
& +\alpha\left\langle\nabla_{\Gamma} \psi, \nabla_{\Gamma} u\right\rangle_{2, \Gamma}+\left\langle\beta \psi+\Upsilon_{2}, u\right\rangle_{2, \Gamma}=\left\langle\Upsilon_{3}, u\right\rangle_{2}, \quad \forall u \in \mathbb{V}_{1} \text {, a.e. in }(0, \infty), \\
& \left\langle\theta_{t}, v\right\rangle_{2}+\langle\nabla \theta, \nabla v\rangle+d\langle\theta, v\rangle_{2, \Gamma}=-\left\langle\Upsilon_{4}, v\right\rangle_{2}, \quad \forall v \in \mathbb{Z}_{K}^{1}, \text { a.e. in }(0, \infty),
\end{aligned}
$$

where we have set

$$
\Upsilon_{1}(t):=f\left(\psi_{1}(t)\right)-f\left(\psi_{2}(t)\right), \quad \Upsilon_{2}:=g\left(\psi_{1}(t)\right)-g\left(\psi_{2}(t)\right),
$$

and

$$
\Upsilon_{3}(t):=\lambda^{\prime}\left(\psi_{1}\right) \theta_{1}(t)-\lambda^{\prime}\left(\psi_{j 2}\right) \theta_{2}(t), \quad \Upsilon_{4}(t):=\lambda^{\prime}\left(\psi_{1}\right)\left(\psi_{1}\right)_{t}-\lambda^{\prime}\left(\psi_{2}\right)\left(\psi_{2}\right)_{t} .
$$

Taking $u=\psi_{t}(t)$ in (2.49), $v=\theta(t)$ in (2.50) and then adding the resulting equations, we deduce that

$$
\begin{aligned}
& \left\|\psi_{t}(t)\right\|_{\mathbb{V}_{0}}^{2}+\|\nabla \theta(t)\|_{2}^{2}+d\left\|\theta_{\mid \Gamma}(t)\right\|_{2, \Gamma}^{2}+\frac{1}{2} \frac{d}{d t}\left[\|\psi(t)\|_{\mathbb{V}_{1}}^{2}+\|\theta(t)\|_{2}^{2}\right] \\
& =-\left\langle\Upsilon_{1}(t), \psi_{t}(t)\right\rangle_{2}-\left\langle\Upsilon_{2}(t), \psi_{t}(t)\right\rangle_{2, \Gamma}+\left\langle\Upsilon_{3}(t), \psi_{t}(t)\right\rangle_{2}-\left\langle\Upsilon_{4}(t), \theta(t)\right\rangle_{2} .
\end{aligned}
$$

We proceed as in [21, Proposition 3]. First, we notice that, thanks to (2.20), we have

$$
\sup _{t \geq 0}\left(\left\|\psi_{i}(t)\right\|_{\mathbb{V}_{1}}^{2}+\left\|\theta_{i}(t)\right\|_{2}^{2}+\int_{0}^{t}\left\|\left(\psi_{i}\right)_{t}(s)\right\|_{\mathbb{V}_{0}}^{2} d s\right) \leq C_{w}, \quad i=1,2,
$$

for some $C_{w}>0$ depending only on the $\mathbb{V}_{1} \times L^{2}(\Omega)$-norm of the initial data. We need to estimate all the remaining terms on the right-hand side of (2.53). To this end, observe preliminarily that, owing to (2.6), we have

$$
\left\{\begin{aligned}
\left|f\left(y_{1}\right)-f\left(y_{2}\right)\right| & \leq c_{f}\left(1+\left|y_{1}\right|^{2}+\left|y_{2}\right|^{2}\right)\left|y_{1}-y_{2}\right| \\
\left|g\left(y_{1}\right)-g\left(y_{2}\right)\right| & \leq c_{g}\left(1+\left|y_{1}\right|^{q}+\left|y_{2}\right|^{q}\right)\left|y_{1}-y_{2}\right|
\end{aligned}\right.
$$

where $q \in[1,+\infty)$ is fixed, but otherwise arbitrary.

In order to estimate the first two terms on the right-hand side of (2.53), we use Young's inequality, Hölder's inequality, (2.51) and (2.55) as follows

$$
\begin{gathered}
\left|\left\langle\Upsilon_{1}(t), \psi_{t}(t)\right\rangle_{2}\right|+\left|\left\langle\Upsilon_{2}(t), \psi_{t}(t)\right\rangle_{2, \Gamma}\right| \\
\leq\left(\left\|\Upsilon_{1}(t)\right\|_{2}^{2}+\left\|\Upsilon_{2}(t)\right\|_{2, \Gamma}^{2}\right)+\frac{1}{4}\left[\left\|\psi_{t}(t)\right\|_{2}^{2}+\left\|\psi_{t}(t)\right\|_{2, \Gamma}^{2}\right] \\
\leq C c_{f}^{2}\left(1+\left\|\psi_{1}(t)\right\|_{6}^{4}+\left\|\psi_{2}(t)\right\|_{6}^{4}\right)\|\psi(t)\|_{6}^{2}
\end{gathered}
$$




$$
+C c_{g}^{2}\left(1+\left\|\psi_{1}(t)\right\|_{3 q, \Gamma}^{2 q}+\left\|\psi_{2}(t)\right\|_{3 q, \Gamma}^{2 q}\right)\|\psi(t)\|_{6, \Gamma}^{2}+\frac{1}{4}\left\|\psi_{t}(t)\right\|_{\mathbb{V}_{0}}^{2} .
$$

Thanks to the continuous embeddings $H^{1}(\Omega) \hookrightarrow L^{6}(\Omega), H^{1}(\Gamma) \hookrightarrow L^{s}(\Gamma)$, for any $s \in$ $[1,+\infty)$, we deduce from $(2.56)$ that

$$
\left|\left\langle\Upsilon_{1}(t), \psi_{t}(t)\right\rangle_{2}\right|+\left|\left\langle\Upsilon_{2}(t), \psi_{t}(t)\right\rangle_{2, \Gamma}\right| \leq C_{w}\|\psi(t)\|_{\mathbb{V}_{1}}^{2}+\frac{1}{4}\left\|\psi_{t}(t)\right\|_{\mathbb{V}_{0}}^{2}
$$

where $C_{w}>0$ depends only on the $\mathbb{V}_{1} \times L^{2}(\Omega)$-norm of the initial data. Moreover, observe that

$$
\begin{aligned}
\left\langle\Upsilon_{3}(t), \psi_{t}(t)\right\rangle_{2}-\left\langle\Upsilon_{4}(t), \theta(t)\right\rangle_{2} & =\left\langle\lambda^{\prime}\left(\psi_{1}\right)-\lambda^{\prime}\left(\psi_{2}\right), \psi_{t}(t) \theta_{2}(t)\right\rangle_{2} \\
& -\left\langle\lambda^{\prime}\left(\psi_{1}\right)-\lambda^{\prime}\left(\psi_{2}\right), \psi_{2 t}(t) \theta(t)\right\rangle_{2} .
\end{aligned}
$$

Then, on account of (2.7), we estimate the last two terms on the right-hand side of (2.53) as follows:

$$
\begin{aligned}
& \left|\left\langle\Upsilon_{3}(t), \psi_{t}(t)\right\rangle_{2}-\left\langle\Upsilon_{4}(t), \theta(t)\right\rangle\right| \\
& \leq C_{\lambda}\left(\left|\left\langle\psi(t) \psi_{t}(t), \theta_{2}(t)\right\rangle_{2}\right|+\left|\left\langle\psi(t),\left(\psi_{2}\right)_{t}(t) \theta(t)\right\rangle_{2}\right|\right) \\
& \leq C_{\lambda}\left(\|\psi(t)\|_{4}\left\|\psi_{t}(t)\right\|_{2}\left\|\theta_{2}(t)\right\|_{4}+\|\psi(t)\|_{4}\|\theta(t)\|_{4}\left\|\left(\psi_{2}\right)_{t}(t)\right\|_{2}\right) \\
& \leq C\|\psi(t)\|_{H^{1}(\Omega)}^{2}\left(\left\|\theta_{2}(t)\right\|_{\mathbb{Z}_{K}^{1}}^{2}+\left\|\left(\psi_{2}\right)_{t}(t)\right\|_{2}^{2}\right)+\frac{1}{4}\left\|\psi_{t}(t)\right\|_{2}^{2}+\frac{1}{2}\|\theta(t)\|_{\mathbb{Z}_{K}^{1}}^{2} .
\end{aligned}
$$

Thus, combining (2.53) with (2.57)-(2.58), we obtain, for $K \in\{D, R\}$,

$$
\begin{gathered}
\frac{1}{2} \frac{d}{d t}\left[\|\psi(t)\|_{\mathbb{V}_{1}}^{2}+\|\theta(t)\|_{2}^{2}\right]+\frac{1}{2}\left\|\psi_{t}(t)\right\|_{2}^{2} \\
+\frac{3}{4}\left\|\psi_{t}(t)\right\|_{2, \Gamma}^{2}+\frac{1}{2}\|\theta(t)\|_{\mathbb{Z}_{K}^{1}}^{2} \leq G_{K}(t)\|\psi(t)\|_{\mathbb{V}_{1}}^{2},
\end{gathered}
$$

where

$$
G_{K}(t):=C_{w}\left(1+\left\|\theta_{2}(t)\right\|_{\mathbb{Z}_{K}^{1}}^{2}+\left\|\left(\psi_{2}\right)_{t}(t)\right\|_{2}^{2}\right) .
$$

It is not difficult to check, on account of (2.20) and (2.54), that $G_{K} \in L^{2}(0, t)$, for all $K \in\{D, N, R\}$ and any fixed $t \geq 0$. The proof of (2.48), in the cases $K \in\{D, R\}$, follows via Gronwall's inequality. It is left to show (2.48) when $K=N$ (we remind that $d=0$ in $(2.53))$. Recall that every weak solution satisfies $\left(\psi_{i}, \theta_{i}\right)$ also satisfies (2.4). Then it easily follows from (2.54) that

$$
\langle\theta(t)\rangle_{\Omega}^{2} \leq C\|\theta(t)\|_{2}^{2}, \quad \forall t \geq 0 .
$$

Adding relation (2.60) to (2.53), then recalling the definition of the norm of $\mathbb{Z}_{N}^{1}$, and arguing as above in (2.59), we immediately obtain estimate (2.48) for $K=N$.

A similar estimate holds for strong solutions. 
Lemma 2.5 Let the assumptions of Theorem 2.3 hold. Consider two global solutions $\left(\psi_{\text {si }}, \theta_{\text {si }}\right)$ to $\mathbf{P}_{\mathbf{K}}^{s}$ corresponding to the initial data $\left(\psi_{0 i}, \theta_{0 i}\right) \in \mathbb{V}_{2} \times \mathbb{Z}_{K}^{1}, i=1,2$. Then, for any $t \geq 0$, the following estimate holds

$$
\begin{gathered}
\left\|\left(\psi_{s 1}-\psi_{s 2}\right)(t)\right\|_{\mathbb{V}_{1}}^{2}+\left\|\left(\theta_{s 1}-\theta_{s 2}\right)(t)\right\|_{2}^{2} \\
+\int_{0}^{t}\left[\left\|\left(\psi_{s 1}-\psi_{s 2}\right)_{t}(s)\right\|_{\mathbb{V}_{0}}^{2}+\left\|\left(\theta_{s 1}-\theta_{s 2}\right)(s)\right\|_{\mathbb{Z}_{K}^{1}}^{2}\right] d s \\
\leq C_{s} e^{L_{s} t}\left(\left\|\psi_{01}-\psi_{02}\right\|_{\mathbb{V}_{1}}^{2}+\left\|\theta_{01}-\theta_{02}\right\|_{2}^{2}\right),
\end{gathered}
$$

where $C_{s}$ and $L_{s}$ are positive constants depending on the norms of the initial data in $\mathbb{V}_{2} \times \mathbb{Z}_{K}^{1}$, on $\Omega$ and on the parameters of the problem, but are both independent of time.

Proof. Here we use the same notation as in the proof of Lemma 2.4. Thanks to (2.40) and (2.55), we have

$$
\left|\Upsilon_{1}(t)\right|+\left|\Upsilon_{2}(t)\right| \leq C_{s}|\psi(t)|, \quad \forall t \geq 0
$$

so that

$$
\begin{aligned}
& \left|\left\langle\Upsilon_{1}(t), \psi_{t}(t)\right\rangle_{2}\right|+\left|\left\langle\Upsilon_{2}(t), \psi_{t}(t)\right\rangle_{2, \Gamma}\right| \\
& \leq C_{s}\left[\|\psi(t)\|_{2}\left\|\psi_{t}(t)\right\|_{2}+\|\psi(t)\|_{2, \Gamma}\left\|\psi_{t}(t)\right\|_{2, \Gamma}\right] \\
& \leq \frac{1}{4}\left\|\psi_{t}(t)\right\|_{\mathbb{V}_{0}}^{2}+C_{s}\|\psi(t)\|_{\mathbb{V}_{0}}^{2} .
\end{aligned}
$$

Furthermore, since estimates (2.58) and (2.60) also hold for strong solutions, combining (2.62) with (2.59), we obtain a similar inequality to (2.59), namely,

$$
\begin{gathered}
\frac{d}{d t}\left[\|\psi(t)\|_{\mathbb{V}_{1}}^{2}+\|\theta(t)\|_{2}^{2}\right]+\frac{1}{2}\left\|\psi_{t}(t)\right\|_{2}^{2} \\
+\frac{3}{4}\left\|\psi_{t}(t)\right\|_{2, \Gamma}^{2}+\frac{1}{2}\|\theta(t)\|_{\mathbb{Z}_{K}^{1}}^{2} \leq L_{s}\|\psi(t)\|_{\mathbb{V}_{1}}^{2},
\end{gathered}
$$

where $L_{s}>0$ depends only on the norm of the initial data in $\mathbb{V}_{2} \times \mathbb{Z}_{K}^{1}$. The proof follows again via Gronwall's inequality.

Straightforward consequences of the above results are the following

Corollary 2.6 Let the assumptions of Theorem 2.2 hold. Then, for each $K \in\{D, N, R\}$, we can define a strongly continuous semigroup

$$
S_{K}^{w}(t): \mathbb{V}_{1} \times L^{2}(\Omega) \rightarrow \mathbb{V}_{1} \times L^{2}(\Omega),
$$

by setting, for all $t \geq 0$,

$$
S_{K}^{w}(t)\left(\psi_{0}, \theta_{0}\right)=\left(\psi_{w}(t), \theta_{w}(t)\right),
$$

where $\left(\psi_{w}, \theta_{w}\right)$ is the unique solution to $\mathbf{P}_{\mathbf{K}}^{w}$. 
Corollary 2.7 Let the assumptions of Theorem 2.3 hold. Then, for each $K \in\{D, N, R\}$, we can define a semigroup

$$
S_{K}^{s}(t): \mathbb{V}_{2} \times \mathbb{Z}_{K}^{1} \rightarrow \mathbb{V}_{2} \times \mathbb{Z}_{K}^{1},
$$

by setting, for all $t \geq 0$,

$$
S_{K}^{s}(t)\left(\psi_{0}, \theta_{0}\right)=\left(\psi_{s}(t), \theta_{s}(t)\right),
$$

where $\left(\psi_{s}, \theta_{s}\right)$ is the unique solution to $\mathbf{P}_{\mathbf{K}}^{s}$. Moreover, $S_{K}^{s}(t)$ is a closed semigroup in the sense of [42].

\section{$3 \quad$ Existence of global attractors}

In this section we want to prove that $S_{K}^{w}(t)$ and $S_{K}^{s}(t)$ have the global attractor. The preliminary step is to prove the dissipativity of the semigroup, that is, the existence of a bounded absorbing set. In the case $K=N$, due to the enthalpy conservation (2.4), we need to put a constraint. More precisely, we set

$$
\begin{aligned}
& \left(\mathbb{V}_{1} \times L^{2}(\Omega)\right)^{M}:=\left\{(v, u) \in \mathbb{V}_{1} \times L^{2}(\Omega):\left|I_{N}(v, u)\right| \leq M\right\}, \\
& \left(\mathbb{V}_{2} \times \mathbb{Z}_{N}^{1}\right)^{M}:=\left\{(v, u) \in \mathbb{V}_{2} \times \mathbb{Z}_{N}^{1}:\left|I_{N}(v, u)\right| \leq M\right\},
\end{aligned}
$$

where $M \geq 0$ is fixed. Note that both the spaces are complete metric spaces with respect to the metrics induced by the norms. Therefore the phase-space of $S_{K}^{w}(t)$ will be

$$
\mathbb{Y}_{0, K}=\left\{\begin{array}{c}
\mathbb{V}_{1} \times L^{2}(\Omega), \quad \text { if } K \in\{D, R\}, \\
\left(\mathbb{V}_{1} \times L^{2}(\Omega)\right)^{M}, \quad \text { if } K=N,
\end{array}\right.
$$

while $S_{K}^{s}(t)$ will act on

$$
\mathbb{Y}_{1, K}=\left\{\begin{array}{l}
\mathbb{V}_{2} \times \mathbb{Z}_{K}^{1}, \quad \text { if } K \in\{D, R\}, \\
\left(\mathbb{V}_{2} \times \mathbb{Z}_{K}^{1}\right)^{M}, \quad \text { if } K=N .
\end{array}\right.
$$

The main results of this section are

Theorem 3.1 Let $f, g, \lambda$ satisfy assumptions (H1)-(H3) and (H5). If $K=N$ suppose in addition that $\lambda$ fulfills (H4). Then $S_{K}^{w}(t)$ possesses the connected global attractor $\mathcal{A}_{K}^{w} \subset \mathbb{Y}_{0, K}$ which is bounded in $\mathbb{V}_{2} \times H^{2}(\Omega)$.

Theorem 3.2 Let $f, g, \lambda$ satisfy assumptions (H1), (H3) and (H5). If $K=N$ suppose in addition that $\lambda$ fulfills (H4). Then $S_{K}^{s}(t)$ possesses the connected global attractor $\mathcal{A}_{K}^{s} \subset \mathbb{Y}_{1, K}$ which is bounded in $\mathbb{V}_{3} \times H^{3}(\Omega)$.

The first proposition is concerned with the existence of a bounded absorbing set in $\mathbb{Y}_{0, K}$ for both the semigroups $S_{K}^{w}(t)$ and $S_{K}^{s}(t)$. 
Lemma 3.3 Let $f, g$ satisfy assumptions (H1) and (H5). Suppose that $\gamma$ satisfies either (H3), if $K \in\{D, R\}$, or (H4), if $K=N$. Then, for any given initial data $\left(\psi_{0}, \theta_{0}\right) \in \mathbb{Y}_{0, K}$, the following estimate holds

$$
\begin{aligned}
& \|(\psi(t), \theta(t))\|_{\mathbb{Y}_{0, K}}^{2}+\int_{t}^{t+1}\left(\left\|\psi_{t}(s)\right\|_{\mathbb{V}_{0}}^{2}+\|\theta(s)\|_{\mathbb{Z}_{K}^{1}}^{2}+\|\psi(s)\|_{L^{4}(\Omega)}^{4}\right) d s \\
& \leq C_{K}\left(\left\|\left(\psi_{0}, \theta_{0}\right)\right\|_{\mathbb{Y}_{0, K}}^{2}+\left\langle F\left(\psi_{0}\right), 1\right\rangle_{2}+\left\langle G\left(\psi_{0}\right), 1\right\rangle_{2, \Gamma}+1\right) e^{-\rho t}+C_{K}^{*},
\end{aligned}
$$

for each $t \geq 0$, where $F$ and $G$ are defined as in (2.19). Here the constants $\rho, C_{K}, C_{K}^{*}$ are independent of $t$ and of the initial data.

Proof. Let us take $u=2 \xi \psi(t)$, for some $\xi>0$, in (2.2). Adding together the obtained relationship with (2.17) and (2.18), we get

$$
\frac{d}{d t} E(t)+\kappa E(t)=\Lambda_{1}(t)
$$

where $\kappa>0$ and

$$
E(t):=\|\psi(t)\|_{\mathbb{V}_{1}}^{2}+2\langle F(\psi(t)), 1\rangle_{2}+2\langle G(\psi(t)), 1\rangle_{2, \Gamma}+\|\theta(t)\|_{2}^{2}+\xi\|\psi(t)\|_{\mathbb{V}_{0}}^{2}+C .
$$

Here the constant $C>0$ is taken large enough in order to ensure that $E(t)$ is nonnegative (recall that $F$ and $G$ are both bounded from below). The function $\Lambda_{1}$ is given by

$$
\begin{aligned}
\Lambda_{1}(t) & :=2 \kappa\left[\langle F(\psi(t))-f(\psi(t)) \psi(t), 1\rangle_{2}+\langle G(\psi(t))-g(\psi(t)) \psi(t), 1\rangle_{2, \Gamma}\right] \\
& -(2 \xi-\kappa)\|\psi(t)\|_{\mathbb{V}_{1}}^{2}-2(\xi-\kappa)\left[\langle f(\psi(t)), \psi(t)\rangle_{2}+\langle g(\psi(t)), \psi(t)\rangle_{2, \Gamma}\right] \\
& -2\left\|\psi_{t}(t)\right\|_{\mathbb{V}_{0}}^{2}-2\|\nabla \theta(t)\|_{2}^{2}-2 d\left\|\theta_{\mid \Gamma}(t)\right\|_{2, \Gamma}^{2} \\
& +\xi \kappa\|\psi(t)\|_{\mathbb{V}_{0}}^{2}+\kappa\|\theta(t)\|_{2}^{2}+\kappa C+2 \xi\left\langle\lambda^{\prime}(\psi(t)) \psi(t), \theta(t)\right\rangle_{2} .
\end{aligned}
$$

Let us first discuss the case when $K \in\{D, R\}$. Observe preliminarily that, owing to (H1), we have

$$
\begin{gathered}
|F(y)| \leq 2 f(y) y+C_{F}, \quad|G(y)| \leq 2 g(y) y+C_{G}, \\
F(y)-f(y) y \leq C_{F}^{\prime}|y|^{2}+C_{F}^{\prime \prime}, \quad G(y)-G(y) y \leq C_{G}^{\prime}|y|^{2}+C_{G}^{\prime \prime},
\end{gathered}
$$

for any $y \in \mathbb{R}$. All the above constants in (3.6)-(3.7) are positive, sufficiently large constants that depend on $F$ and $G$, only. Moreover, using assumption (H3), standard Hölder's and Young's inequalities, we estimate the last term on the right-hand side of (3.5) as follows

$$
\begin{aligned}
2 \xi\left|\left\langle\lambda^{\prime}(\psi) \psi, \theta\right\rangle_{2}\right| & \leq 2 \xi c_{\lambda}\langle|\psi|,|\theta|\rangle_{2}+2 \xi c_{\lambda}\left\langle|\psi|^{2},|\theta|\right\rangle_{2} \\
& \leq\|\theta\|_{\mathbb{Z}_{K}^{1}}^{2}+2 \xi^{2} c_{\lambda}^{2}\left(\|\psi\|_{\mathbb{V}_{0}}^{2}+\|\psi\|_{L^{4}(\Omega)}^{4}\right) .
\end{aligned}
$$


From (3.6)-(3.8) and assumption (H5), it follows

$$
\begin{aligned}
\Lambda_{1}(t) \leq & -\left(2 \xi-\kappa-2 \kappa\left(C_{F}^{\prime}+C_{G}^{\prime}\right)-\xi \kappa-\xi^{2} c\right)\|\psi(t)\|_{\mathbb{V}_{1}}^{2}-2\left\|\psi_{t}(t)\right\|_{\mathbb{V}_{0}}^{2} \\
& -\frac{(\xi-\kappa)}{2}\langle|F(\psi(t))|, 1\rangle_{2}-(\xi-\kappa)\langle|G(\psi(t))|, 1\rangle_{2, \Gamma} \\
- & \left(1-\kappa C_{0}\right)\|\theta\|_{\mathbb{Z}_{K}^{1}}^{2}-\left[\xi\left(\eta_{1}-c \xi\right)-\kappa \eta_{1}\right]\|\psi(t)\|_{L^{4}(\Omega)}^{4}+C_{1},
\end{aligned}
$$

where $C_{0}>0$ and $C_{1}>0$ depends on $\xi, \kappa$ and $C_{F}^{\prime \prime}$, $C_{G}^{\prime \prime}$ at most and $c>0$ depends only on $c_{\lambda}$. From now on $C_{i}$ stands for a positive constant which is independent on the initial data and on time.

It is thus possible to adjust $\xi<1$ small enough and $\kappa \in(0, \xi)$ (possibly even smaller than $\xi^{2}$ ) in order to have

$$
\begin{aligned}
\frac{d}{d t} E(t)+\kappa E(t) & +C_{2}\left(\|\psi(t)\|_{\mathbb{V}_{1}}^{2}+\left\|\psi_{t}(t)\right\|_{\mathbb{V}_{0}}^{2}+\|\theta(t)\|_{\mathbb{Z}_{K}^{1}}^{2}+\|\psi(t)\|_{L^{4}(\Omega)}^{4}\right) \\
& +C_{3}\left(\|F(\psi(t))\|_{1}+\|G(\psi(t))\|_{1, \Gamma}\right) \leq C_{1}
\end{aligned}
$$

from which we deduce that

$$
\frac{d}{d t} E(t)+\kappa E(t) \leq C_{1} .
$$

On the other hand, one can check that there exists a positive constant $C_{4}$, independent of $t$ and on the initial data, such that

$$
\|(\psi(t), \theta(t))\|_{\mathbb{Y}_{0, K}}^{2} \leq C_{4} E(t), \forall t \geq 0 .
$$

Then, applying Gronwall's lemma to (3.10) we deduce that

$$
E(t) \leq C_{5} E(0) e^{-\kappa t}+C_{6}, \quad \forall t \geq 0 .
$$

Integrating (3.9) over $(t, t+1)$ and employing estimate (3.12) we obtain

$$
\begin{aligned}
& E(t)+\int_{t}^{t+1}\left(\|\psi(s)\|_{\mathbb{V}_{1}}^{2}+\left\|\psi_{t}(s)\right\|_{\mathbb{V}_{0}}^{2}+\|\theta(s)\|_{\mathbb{Z}_{K}^{1}}^{2}\right) d s \\
& +\int_{t}^{t+1}\left(\|F(\psi(s))\|_{1}+\|G(\psi(s))\|_{1, \Gamma}+\|\psi(s)\|_{L^{4}(\Omega)}^{4}\right) d s \\
& \leq C_{7} E(0) e^{-\kappa t}+C_{8}, \quad \forall t \geq 0 .
\end{aligned}
$$

Finally, on account of (3.11), we easily obtain the required estimate (3.3) for $K \in\{D, R\}$.

To prove a similar uniform inequality when $K=N$ we need to use the enthalpy conservation (cf. (2.4)). Observe that

$$
\begin{aligned}
2 \xi\left\langle\lambda^{\prime}(\psi) \psi, \theta\right\rangle_{2} & =2 \xi\left\langle\theta-\langle\theta\rangle_{\Omega}, \lambda^{\prime}(\psi) \psi\right\rangle_{2}+2 \xi|\Omega| I_{N}\left(\psi_{0}, \theta_{0}\right)\left\langle\lambda^{\prime}(\psi) \psi\right\rangle_{\Omega} \\
& -2 \xi|\Omega|\langle\lambda(\psi)\rangle_{\Omega}\left\langle\lambda^{\prime}(\psi) \psi\right\rangle_{\Omega} .
\end{aligned}
$$


Employing a standard Poincaré's inequality, we know that

$$
\left\|v-\langle v\rangle_{\Omega}\right\|_{2}^{2}=\|v\|_{2}^{2}-|\Omega|\langle v\rangle_{\Omega}^{2} \leq C_{\Omega}\|\nabla v\|_{2}^{2}
$$

for some $C_{\Omega}>0$.

Let us rewrite $\Lambda_{1}$ defined by (3.5), taking (3.14) into account. We obtain

$$
\begin{aligned}
\Lambda_{1}(t) & :=2 \kappa\left[\langle F(\psi(t))-f(\psi(t)) \psi(t), 1\rangle_{2}+\langle G(\psi(t))-g(\psi(t)) \psi(t), 1\rangle_{2, \Gamma}\right] \\
& -(2 \xi-\kappa)\|\psi(t)\|_{\mathbb{V}_{1}}^{2}-2(\xi-\kappa)\left[\langle f(\psi(t)), \psi(t)\rangle_{2}+\langle g(\psi(t)), \psi(t)\rangle_{2, \Gamma}\right] \\
& -2\left\|\psi_{t}(t)\right\|_{\mathbb{V}_{0}}^{2}-2\|\nabla \theta(t)\|_{2}^{2}+2 \xi \kappa\|\psi(t)\|_{\mathbb{V}_{0}}^{2}+\kappa\|\theta(t)\|_{2}^{2} \\
& +\kappa C+2 \xi\left\langle\theta(t)-\langle\theta(t)\rangle_{\Omega}, \lambda^{\prime}(\psi(t)) \psi(t)\right\rangle_{2} \\
& +2 \xi|\Omega| I_{N}\left(\psi_{0}, \theta_{0}\right)\left\langle\lambda^{\prime}(\psi(t)) \psi(t)\right\rangle_{\Omega}-2 \xi|\Omega|\langle\lambda(\psi)\rangle_{\Omega}\left\langle\lambda^{\prime}(\psi(t)) \psi(t)\right\rangle_{\Omega} .
\end{aligned}
$$

It is worth mentioning that, from (2.4), it is also not too difficult to deduce the following estimate:

$$
\begin{aligned}
\kappa\|\theta\|_{2}^{2} & =\kappa\left\|\left(\theta-\langle\theta\rangle_{\Omega}\right)+\langle\theta\rangle_{\Omega}\right\|_{2}^{2} \\
& \leq 2 \kappa\left\|\theta-\langle\theta\rangle_{\Omega}\right\|_{2}^{2}+2 \kappa|\Omega|\left(I_{N}\left(\psi_{0}, \theta_{0}\right)-\langle\lambda(\psi)\rangle_{\Omega}\right)^{2} \\
& \leq 2 \kappa\left\|\theta-\langle\theta\rangle_{\Omega}\right\|_{2}^{2}+\kappa c\|\psi\|_{2}^{2}+\kappa c\|\psi\|_{L^{4}(\Omega)}^{4}+C_{M},
\end{aligned}
$$

where the constant $c>0$ is independent of $t, \xi, \kappa, M$ and initial data, and $C_{M}>0$ depends on $M, \xi, \kappa$ and $\Omega$, but is independent of time and initial data. From now on, $c$ will stand for a positive constant having these properties. It remains to estimate all the terms on the right-hand side of (3.14). We begin by estimating the first term, arguing exactly as in (3.8). Recalling (H4), from (3.15) we obtain that

$$
\begin{aligned}
2 \xi\left|\left\langle\theta-\langle\theta\rangle_{\Omega}, \lambda^{\prime}(\psi) \psi\right\rangle_{2}\right| & \leq 2 \xi C_{\gamma}\left\langle\left|\theta-\langle\theta\rangle_{\Omega}\right|,|\psi|\right\rangle_{2}+4 a \xi\left\langle\left|\theta-\langle\theta\rangle_{\Omega}\right|, \psi^{2}\right\rangle_{2} \\
& \leq\|\nabla \theta\|_{2}^{2}+c \xi^{2}\|\psi\|_{\mathbb{V}_{0}}^{2}+c \xi^{2}\|\psi\|_{L^{4}(\Omega)}^{4} .
\end{aligned}
$$

On the other hand,

$$
\begin{aligned}
2 \xi|\Omega| I_{N}\left(\psi_{0}, \theta_{0}\right)\left\langle\lambda^{\prime}(\psi) \psi\right\rangle_{\Omega} & =2 \xi|\Omega| I_{N}\left(\psi_{0}, \theta_{0}\right)\left\langle\gamma^{\prime}(\psi) \psi\right\rangle_{\Omega}-4 a \xi|\Omega| I_{N}\left(\psi_{0}, \theta_{0}\right)\left\langle\psi^{2}\right\rangle_{\Omega} \\
& \leq \xi^{2}\|\psi\|_{2}^{2}+\xi^{2}\|\psi\|_{L^{4}(\Omega)}^{4}+C_{M},
\end{aligned}
$$

where the positive constant $C_{M}$ depends clearly on $M$. Finally, it is not difficult to show, using standard Hölder's and Young's inequalities, that

$$
\begin{aligned}
-2 \xi|\Omega|\langle\lambda(\psi)\rangle_{\Omega}\left\langle\lambda^{\prime}(\psi) \psi\right\rangle_{\Omega} & =-4 \xi|\Omega| a^{2}\left\langle\psi^{2}\right\rangle_{\Omega}^{2}-2 \xi|\Omega|\langle\gamma(\psi)\rangle_{\Omega}\left\langle\gamma^{\prime}(\psi) \psi\right\rangle_{\Omega} \\
& +4 a \xi|\Omega|\langle\gamma(\psi)\rangle_{\Omega}\left\langle\psi^{2}\right\rangle_{\Omega}+2 a \xi|\Omega|\left\langle\psi^{2}\right\rangle_{\Omega}\left\langle\gamma^{\prime}(\psi) \psi\right\rangle_{\Omega} \\
& \leq-4 \xi|\Omega| a^{2}\left\langle\psi^{2}\right\rangle_{\Omega}^{2}+\left(\xi^{2}+\xi^{4 / 3}\right)\|\psi\|_{4}^{4}+C_{9} .
\end{aligned}
$$


Combining estimates (3.18)-(3.20) together, it easily follows that

$$
\begin{aligned}
2 \xi\left\langle\lambda^{\prime}(\psi) \psi, \theta\right\rangle_{2} & \leq-4 \xi|\Omega| a^{2}\left\langle\psi^{2}\right\rangle_{\Omega}^{2}+\|\nabla \theta\|_{2}^{2}+\xi^{2}(c+1)\|\psi\|_{2}^{2} \\
& +\left[(1+c) \xi^{2}+\xi^{4 / 3}\right]\|\psi\|_{L^{4}(\Omega)}^{4}+C_{M}^{\prime},
\end{aligned}
$$

for a new positive constant $C_{M}^{\prime}$. Using (3.17), (3.6)-(3.7), (3.15) and (3.21), we infer from $(3.16)$

$$
\begin{aligned}
\Lambda_{1}(t) & \leq-\left[2 \xi-\kappa(1+c)-2 \kappa\left(C_{F}^{\prime}+C_{G}^{\prime}\right)-2 \xi \kappa-\xi^{2}(c+1)\right]\|\psi(t)\|_{\mathbb{V}_{1}}^{2} \\
& -2\left\|\psi_{t}(t)\right\|_{\mathbb{V}_{0}}^{2}-\frac{(\xi-\kappa)}{2}\langle|F(\psi(t))|, 1\rangle_{2}-(\xi-\kappa)\langle|G(\psi(t))|, 1\rangle_{2, \Gamma} \\
& -\left(1-2 \kappa C_{\Omega}\right)\|\nabla \theta(t)\|_{2}^{2}-4 \xi|\Omega| a^{2}\left\langle\psi^{2}\right\rangle_{\Omega}^{2} \\
& -\left(\xi\left[\eta_{1}-(1+c) \xi+\xi^{1 / 3}\right]-\kappa\left(\eta_{1}+c\right)\right)\|\psi(t)\|_{L^{4}(\Omega)}^{4}+C_{10},
\end{aligned}
$$

where $C_{10}>0$ depends on $M, \xi, \kappa$ and $\Omega$, but is independent of time and initial data. It is thus possible to adjust $\xi<1$ small enough and $\kappa \in(0, \xi)$ in order to deduce (cf. (3.4), $(3.11),(3.22))$

$$
\frac{d}{d t} E(t)+\kappa E(t)+\kappa^{\prime}\left(\|\psi(t)\|_{\mathbb{V}_{1}}^{2}+\|\psi(t)\|_{L^{4}(\Omega)}^{4}\right)+\kappa^{\prime \prime}\left(\left\|\psi_{t}(t)\right\|_{\mathbb{V}_{0}}^{2}+\|\nabla \theta(t)\|_{2}^{2}\right) \leq C_{11}
$$

Applying a suitable version of Gronwall's inequality to (3.23) and taking (3.11) into account, we obtain the analogue of estimate (3.3) in the case $K=N$, except for the $L^{2}\left([t, t+1] ; H^{1}\right)$ norm of $\theta$. More precisely, we obtain

$$
\begin{aligned}
& E(t)+\int_{t}^{t+1}\left(\|\psi(s)\|_{\mathbb{V}_{1}}^{2}+\|\psi(t)\|_{L^{4}(\Omega)}^{4}+\left\|\psi_{t}(s)\right\|_{\mathbb{V}_{0}}^{2}+\|\nabla \theta(s)\|_{2}^{2}\right) d s \\
& \leq C_{12} E(0) e^{-\kappa t}+C_{13}, \quad \forall t \geq 0 .
\end{aligned}
$$

However, recalling (2.21) we easily obtain estimate (3.3) also for $K=N$. The proof is finished.

We now show the existence of a compact absorbing set, namely,

Lemma 3.4 Let the assumptions of Theorem 3.1 be satisfied. Then, there is a positive nondecreasing monotone function $Q$ and, for any $R_{0}>0$, there exists $t_{0}=t_{0}\left(R_{0}\right)>0$ such that

$$
\left\|S_{K}^{w}(t)\left(\psi_{0}, \theta_{0}\right)\right\|_{\mathbb{V}_{2} \times H^{2}(\Omega)} \leq Q\left(R_{0}\right), \quad \forall t \geq t_{0},
$$

for any $\left(\psi_{0}, \theta_{0}\right) \in \mathcal{B}\left(R_{0}\right) \subset \mathbb{Y}_{0, K}$, where $\mathcal{B}\left(R_{0}\right)$ is a ball of radius $R_{0}$, centered at 0 . 
Proof. We recall that all the following formal arguments can be justified by recalling that the existence of a smooth local solution can be proven (see [20, Section 3]). Recall first that (2.33) entails

$$
\begin{aligned}
& \frac{d}{d t}\left(\left\|\psi_{t}(t)\right\|_{2}^{2}+\left\|\psi_{t}(t)\right\|_{2, \Gamma}^{2}+\|\nabla \theta(t)\|_{2}^{2}+d\left\|\theta_{\mid \Gamma}(t)\right\|_{2, \Gamma}^{2}\right) \\
& +\left\|\psi_{t}(t)\right\|_{2}^{2}+\left\|\psi_{t}(t)\right\|_{2, \Gamma}^{2}+\|\nabla \theta(t)\|_{2}^{2}+d\left\|\theta_{\mid \Gamma}(t)\right\|_{2, \Gamma}^{2} \\
& +2\left\|\psi_{t}(t)\right\|_{\mathbb{V}_{1}}^{2}+2\left\|\theta_{t}(t)\right\|_{2}^{2}=\Lambda_{2}(t),
\end{aligned}
$$

where

$$
\begin{aligned}
\Lambda_{2}(t) & :=-2\left\langle f^{\prime}(\psi(t)), \psi_{t}^{2}(t)\right\rangle_{2}-2\left\langle g^{\prime}(\psi(t)), \psi_{t}^{2}(t)\right\rangle_{2, \Gamma} \\
& +\left\|\psi_{t}(t)\right\|_{2}^{2}+\left\|\psi_{t}(t)\right\|_{2, \Gamma}^{2}+\|\nabla \theta(t)\|_{2}^{2}+d\left\|\theta_{\mid \Gamma}(t)\right\|_{2, \Gamma}^{2} \\
& +\left\langle\lambda^{\prime \prime}(\psi(t)) \psi_{t}^{2}(t), \theta(t)\right\rangle_{2} .
\end{aligned}
$$

Recalling (2.34)-(2.35) we have that

$$
\begin{aligned}
& \frac{d}{d t}\left(\left\|\psi_{t}(t)\right\|_{2}^{2}+\left\|\psi_{t}(t)\right\|_{2, \Gamma}^{2}+\|\nabla \theta(t)\|_{2}^{2}+d\left\|\theta_{\mid \Gamma}(t)\right\|_{2, \Gamma}^{2}\right) \\
& +\left\|\psi_{t}(t)\right\|_{2}^{2}+\left\|\psi_{t}(t)\right\|_{2, \Gamma}^{2}+\|\nabla \theta(t)\|_{2}^{2}+d\left\|\theta_{\mid \Gamma}(t)\right\|_{2, \Gamma}^{2} \\
& +2\left\|\psi_{t}(t)\right\|_{\mathbb{V}_{1}}^{2}+2\left\|\theta_{t}(t)\right\|_{2}^{2} \\
& \leq C\left\|\psi_{t}(t)\right\|_{\mathbb{V}_{0}}^{2}+\left\|\psi_{t}(t)\right\|_{2}^{2}+\left\|\psi_{t}(t)\right\|_{2, \Gamma}^{2}+\|\nabla \theta(t)\|_{2}^{2}+d\left\|\theta_{\mid \Gamma}(t)\right\|_{2, \Gamma}^{2} \\
& +\eta\left(\left\|\nabla \psi_{t}\right\|_{2}^{2}+\left\|\psi_{t}\right\|_{2}^{2}\right)+C_{\eta}\left\|\psi_{t}\right\|_{2}^{2}\|\theta\|_{2}^{4} .
\end{aligned}
$$

Since $\eta$ is small (cf. (2.35)), then we get

$$
\begin{aligned}
& \frac{d}{d t}\left(\left\|\psi_{t}(t)\right\|_{2}^{2}+\left\|\psi_{t}(t)\right\|_{2, \Gamma}^{2}+\|\nabla \theta(t)\|_{2}^{2}+d\left\|\theta_{\mid \Gamma}(t)\right\|_{2, \Gamma}^{2}\right) \\
& +\left\|\psi_{t}(t)\right\|_{2}^{2}+\left\|\psi_{t}(t)\right\|_{2, \Gamma}^{2}+\|\nabla \theta(t)\|_{2}^{2}+d\left\|\theta_{\mid \Gamma}(t)\right\|_{2, \Gamma}^{2} \\
& +C_{1}\left(\left\|\psi_{t}(t)\right\|_{\mathbb{V}_{1}}^{2}+\left\|\theta_{t}(t)\right\|_{2}^{2}\right) \\
& \leq C_{2}\left(\left\|\psi_{t}(t)\right\|_{\mathbb{V}_{0}}^{2}+\|\theta(t)\|_{\mathbb{Z}_{K}^{1}}^{2}+\left\|\psi_{t}(t)\right\|_{2}^{2}\|\theta(t)\|_{2}^{4}\right)=: \Lambda_{3}(t) .
\end{aligned}
$$

Due to (3.3) we easily deduce that

$$
\begin{aligned}
& \sup _{t \geq 0} \int_{t}^{t+1}\left|\Lambda_{3}(s)\right| d s \\
& \leq C \sup _{t \geq 0} \int_{t}^{t+1}\left(\left\|\psi_{t}(s)\right\|_{\mathbb{V}_{0}}^{2}+\|\theta(s)\|_{\mathbb{Z}_{K}^{1}}^{2}\right) d s+C \sup _{t \geq 0}\|\theta(t)\|_{2}^{4} \sup _{t \geq 0} \int_{t}^{t+1}\left\|\psi_{t}(s)\right\|_{2}^{2} d s \\
& \leq Q\left(\left\|\left(\psi_{0}, \theta_{0}\right)\right\|_{\mathbb{Y}_{0, K}}^{2}+\left\langle F\left(\psi_{0}\right), 1\right\rangle_{2}+\left\langle G\left(\psi_{0}\right), 1\right\rangle_{2, \Gamma}+1\right) e^{-\rho t}+C .
\end{aligned}
$$


Here and in the sequel $C$ stands for a positive constant that is independent of $t$ and of the initial data. This constant may vary from line to line. Besides, $Q$ denotes a positive nondecreasing monotone function which is independent of $t$. Using the so-called uniform Gronwall lemma (see, e.g., [47]), on account of (3.29), from (3.28) and the hypothesis of the lemma, we infer the existence of $t_{0}>0$ such that

$$
\begin{aligned}
& \left\|\psi_{t}(t)\right\|_{2}^{2}+\left\|\psi_{t}(t)\right\|_{2, \Gamma}^{2}+\|\theta(t)\|_{\mathbb{Z}_{K}^{1}}^{2}+\int_{t}^{t+1}\left[\left\|\psi_{t}(s)\right\|_{\mathbb{V}_{1}}^{2}+\left\|\theta_{t}(s)\right\|_{2}^{2}\right] d s \\
& \leq Q\left(\left\|\left(\psi_{0}, \theta_{0}\right)\right\|_{\mathbb{Y}_{m, K}}^{2}\right) e^{-\rho t}+C, \quad \forall t \geq t_{0} .
\end{aligned}
$$

Applying now the maximum principle to the boundary value problem (2.38), as in the proof of Theorem 2.2, we infer from (2.39), (3.3) and (3.30) that

$$
\begin{aligned}
\|\psi(t)\|_{\infty}^{2}+\|\psi(t)\|_{\infty, \Gamma}^{2} & \leq C\left(1+\left\|h_{1}(t)\right\|_{2}^{2}+\left\|h_{2}(t)\right\|_{2, \Gamma}^{2}\right) \\
& \leq Q\left(\left\|\left(\psi_{0}, \theta_{0}\right)\right\|_{\mathbb{Y}_{m, K}}^{2}\right) e^{-\rho t}+C, \quad \forall t \geq t_{0} .
\end{aligned}
$$

In order to get the estimate for $\psi(t)$ in $\mathbb{V}_{2}$, we rewrite problem (2.38) into the form

$$
\left\{\begin{array}{c}
-\Delta \psi=h_{3}:=-f(\psi)+\lambda^{\prime}(\psi) \theta-\psi_{t}, \quad \text { in } \Omega, \text { a.e. in }\left(t_{0},+\infty\right), \\
-\alpha \Delta_{\Gamma} \psi+\partial_{\mathbf{n}} \psi+\beta \psi=h_{4}:=-\psi_{t}-g(\psi), \quad \text { on } \Gamma, \text { a.e. in }\left(t_{0},+\infty\right),
\end{array}\right.
$$

and apply the result of $[20$, Lemma $2.2,(2.11)]$. We obtain

$$
\begin{aligned}
\|\psi(t)\|_{\mathbb{V}_{2}}^{2} & \leq C\left(\left\|h_{3}(t)\right\|_{2}^{2}+\left\|h_{4}(t)\right\|_{2, \Gamma}^{2}\right) \\
& \leq C\left(\|f(\psi(t))\|_{2}^{2}+\|g(\psi(t))\|_{2, \Gamma}^{2}+\|\theta(t)\|_{2}^{2}+\|\psi(t)\|_{\mathbb{V}_{1}}^{2}+\left\|\psi_{t}(t)\right\|_{\mathbb{V}_{0}}^{2}\right),
\end{aligned}
$$

which yields, on account of (3.3), (3.30) and (3.31), the following estimate:

$$
\|\psi(t)\|_{\mathbb{V}_{2}} \leq Q\left(R_{0}\right), \quad \forall t \geq t_{0} .
$$

The proof is thus complete.

Lemma 3.5 Let the assumptions of Theorem 3.2 be satisfied. Then, there is a positive nondecreasing monotone function $Q$ and, for any $R_{1}>0$, there exists $t_{1}=t_{1}\left(R_{1}\right)>0$ such that

$$
\left\|S_{K}^{s}(t)\left(\psi_{0}, \theta_{0}\right)\right\|_{\mathbb{V}_{3} \times H^{3}(\Omega)} \leq Q\left(R_{1}\right), \quad \forall t \geq t_{1}
$$

for any $\left(\psi_{0}, \theta_{0}\right) \in \mathcal{B}\left(R_{1}\right) \subset \mathbb{Y}_{1, K}$, where $\mathcal{B}\left(R_{1}\right)$ is a ball of radius $R_{1}$, centered at 0 .

Proof. Observe first that, on account of Lemmas 3.3 and 3.4, it is easy to realize that $S_{K}^{s}(t)$ has a bounded absorbing set in $\mathbb{Y}_{1, K}$. Thus, without loss of generality, we assume that $\mathcal{B}\left(R_{1}\right)$ is such a bounded absorbing set. 
We need to obtain higher-order estimates. Even in this case we will proceed formally recalling [20, Theorem 5, Section 3]. Let us differentiate (2.2) and (2.3) with respect to time. Then, we take $u=\psi_{t t}(t)$ and by $v=\theta_{t}(t)$, respectively. Adding up the resulting relations, we obtain

$$
\begin{aligned}
& \frac{1}{2} \frac{d}{d t}\left[\left\|\nabla \psi_{t}(t)\right\|_{2}^{2}+\alpha\left\|\nabla_{\Gamma} \psi_{t}(t)\right\|_{2, \Gamma}^{2}+\beta\left\|\psi_{t}(t)\right\|_{2, \Gamma}^{2}+\left\|\theta_{t}(t)\right\|_{2}^{2}\right] \\
& +\left\|\psi_{t t}(t)\right\|_{2}^{2}+\left\|\psi_{t t}(t)\right\|_{2, \Gamma}^{2}+\left\|\nabla \theta_{t}(t)\right\|_{2}^{2}+d\left\|\left(\theta_{\mid \Gamma}\right)_{t}(t)\right\|_{2, \Gamma}^{2} \\
& =-\int_{\Omega} f^{\prime}(\psi(t)) \psi_{t}(t) \psi_{t t}(t) d x-\int_{\Gamma} g^{\prime}(\psi(t)) \psi_{t}(t) \psi_{t t}(t) d S \\
& +\left\langle\lambda^{\prime \prime}(\psi(t)) \psi_{t}(t), \psi_{t t}(t) \theta(t)\right\rangle_{2}-\left\langle\lambda^{\prime \prime}(\psi(t)), \psi_{t}^{2}(t) \theta_{t}(t)\right\rangle_{2} .
\end{aligned}
$$

Using Hölder's and Young's inequalities to estimate the last two terms on the right-hand side of equality (3.35), exactly as in (2.35), we have, for $t \geq 0$,

$$
\begin{aligned}
& \frac{d}{d t} \mathcal{Y}(t)+\left\|\psi_{t t}(t)\right\|_{\mathbb{V}_{0}}^{2}+2\left(\left\|\nabla \theta_{t}(t)\right\|_{2}^{2}+d\left\|\left(\theta_{\mid \Gamma}\right)_{t}(t)\right\|_{2, \Gamma}^{2}\right) \\
& \leq H_{1}(t) \mathcal{Y}(t)+H_{2}(t),
\end{aligned}
$$

where we have set

$$
\mathcal{Y}(t):=\left\|\psi_{t}(t)\right\|_{\mathbb{V}_{1}}^{2}+\left\|\theta_{t}(t)\right\|_{2}^{2},
$$

and

$$
\begin{aligned}
& H_{1}(t):=C\left(\left\|\psi_{t}(t)\right\|_{2}^{2}\left\|\theta_{t}(t)\right\|_{2}^{2}\right), \\
& H_{2}(t):=C\left(\left\|f^{\prime}(\psi(t)) \psi_{t}(t)\right\|_{2}^{2}+\left\|g^{\prime}(\psi(t)) \psi_{t}(t)\right\|_{2, \Gamma}^{2}+\left\|\psi_{t}(t)\right\|_{H^{1}(\Omega)}^{2}+\left\|\psi_{t}(t)\right\|_{2}^{2}\|\theta(t)\|_{2}^{4}\right) .
\end{aligned}
$$

Observe now that, owing to (3.30) and (3.31), we infer that

$$
\sup _{t \geq 0} \int_{t}^{t+1} H_{1}(s) d s \leq Q\left(R_{1}\right) \text { and } \sup _{t \geq 0} \int_{t}^{t+1} H_{2}(s) d s \leq Q\left(R_{1}\right) .
$$

Then, using (3.37), we can apply to (3.36) the uniform Gronwall lemma once more and find $t_{*}>0$, depending on $R_{1}$, such that

$$
\left\|\psi_{t}(t)\right\|_{\mathbb{V}_{1}}^{2}+\left\|\theta_{t}(t)\right\|_{2}^{2}+\int_{t}^{t+1}\left\|\psi_{t t}(s)\right\|_{\mathbb{V}_{0}}^{2} d s \leq Q\left(R_{1}\right), \quad \forall t \geq t_{*} .
$$

Let us consider again equation (2.3) differentiated with respect to $t$ and take $v=\theta_{t t}(t)$. Then we get

$$
\begin{aligned}
& \left\|\theta_{t t}(t)\right\|_{2}^{2}+\frac{1}{2} \frac{d}{d t}\left(\left\|\nabla \theta_{t}(t)\right\|_{2}^{2}+d\left\|\theta_{t \mid \Gamma}(t)\right\|_{2, \Gamma}^{2}\right) \\
& =-\left\langle\lambda^{\prime \prime}(\psi) \psi_{t}^{2}(t), \theta_{t t}(t)\right\rangle_{2}-\left\langle\lambda^{\prime}(\psi) \psi_{t t}(t), \theta_{t t}(t)\right\rangle_{2} .
\end{aligned}
$$


which yields, via standard Sobolev inequalities,

$$
\begin{aligned}
& \frac{1}{2}\left\|\theta_{t t}(t)\right\|_{2}^{2}+\frac{1}{2} \frac{d}{d t}\left(\left\|\nabla \theta_{t}(t)\right\|_{2}^{2}+d\left\|\theta_{t \mid \Gamma}(t)\right\|_{2, \Gamma}^{2}\right) \\
& \leq C\left\|\psi_{t}(t)\right\|_{H^{1}(\Omega)}^{4}+Q\left(\|\psi(t)\|_{\infty}\right)\left\|\psi_{t t}(t)\right\|_{2}^{2} .
\end{aligned}
$$

From (3.31), (3.36) and (3.38) we infer that

$$
\sup _{t \geq t_{1}} \int_{t}^{t+1}\left(\left\|\nabla \theta_{t}(s)\right\|_{2}^{2}+d\left\|\left(\theta_{\mid \Gamma}\right)_{t}(s)\right\|_{2, \Gamma}^{2}\right) d s \leq Q\left(R_{1}\right)
$$

and

$$
\sup _{t \geq t_{1}} \int_{t}^{t+1}\left(\left\|\psi_{t}(s)\right\|_{H^{1}(\Omega)}^{4}+Q\left(\|\psi(s)\|_{\infty}\right)\left\|\psi_{t t}(s)\right\|_{2}^{2}\right) d s \leq Q\left(R_{1}\right) .
$$

We can now apply the uniform Gronwall lemma once again and infer from (3.39) the existence of $t_{1} \geq t_{*}$ such that

$$
\left\|\nabla \theta_{t}(t)\right\|_{2}^{2}+d\left\|\theta_{t \mid \Gamma}(t)\right\|_{2, \Gamma}^{2} \leq Q\left(R_{1}\right), \quad \forall t \geq t_{1}
$$

Therefore, (3.38) and (3.40) allow us to deduce via standard elliptic regularity, the following bound

$$
\|\theta(t)\|_{H^{3}(\Omega)} \leq Q\left(R_{1}\right), \quad K \in\{D, N, R\}
$$

for each $t \geq t_{1}$.

Furthermore, with the help of estimates (3.31), (3.33) and (3.38), we also obtain from [39, Corollary A.1] (see also [20, Lemma 2.2])

$$
\begin{gathered}
\|\psi(t)\|_{\mathbb{V}_{3}}^{2} \leq C\left(\|f(\psi(t))\|_{H^{1}(\Omega)}^{2}+\|g(\psi(t))\|_{H^{1}(\Gamma)}^{2}\right. \\
\left.+Q\left(\|\psi(t)\|_{\infty}\right)\|\theta(t)\|_{H^{1}(\Omega)}^{2}+\|\psi(t)\|_{\mathbb{V}_{2}}^{2}+\left\|\psi_{t}(t)\right\|_{\mathbb{V}_{1}}^{2}\right),
\end{gathered}
$$

which yields, on account of the above estimates,

$$
\|\psi(t)\|_{\mathbb{V}_{3}}^{2} \leq Q\left(R_{0}\right), \quad \forall t \geq t_{1} .
$$

Summing up, we conclude by observing that (3.34) follows from (3.41) and (3.43).

Proof of Theorem 3.1. On account of Corollary 2.6, Lemma 3.3 and Lemma 3.4 the proof follows from well-known general results (see, e.g., [47]).

Proof of Theorem 3.2. Thanks to Lemma 3.5 the dynamical systems $\left(\mathbb{Y}_{1, K}, S_{K}^{s}(t)\right)$ has an absorbing set in $\mathbb{Y}_{1, K}$ which is bounded in $\mathbb{V}_{3} \times H^{3}(\Omega)$. Moreover, recalling Corollary 2.7, we have that $S_{K}^{s}(t)$ is also a closed semigroup. Thus the proof follows from [42, Theorem 2 and Corollary 6]. 


\section{Existence of exponential attractors}

We begin to prove the following

Theorem 4.1 Let $f, g \in C^{2}(\mathbb{R})$ and $\lambda \in C^{3}(\mathbb{R})$ satisfy the assumptions of Theorem 3.1. Then, $S_{K}^{w}(t)$ possesses an exponential attractor $\mathcal{M}_{K}^{w}$, bounded in $\mathbb{V}_{2} \times H^{2}(\Omega)$, namely,

$(I) \mathcal{M}_{K}^{w}$ is compact and positively invariant with respect $S_{K}^{w}(t)$, i.e.,

$$
S_{K}^{w}(t)\left(\mathcal{M}_{K}^{w}\right) \subset \mathcal{M}_{K}^{w}, \quad \forall t \geq 0
$$

(II) The fractal dimension of $\mathcal{M}_{K}^{w}$ with respect to the $\mathbb{Y}_{0, K}$-metric is finite.

(III) There exist a positive nondecreasing monotone function $Q_{w}$ and a constant $\rho_{w}>0$ such that

$$
\operatorname{dist}_{\mathbb{Y}_{0, K}}\left(S_{K}^{w}(t) B, \mathcal{M}_{K}^{w}\right) \leq Q_{w}\left(\|B\|_{\mathbb{Y}_{0, K}}\right) e^{-\rho_{w} t}, \quad \forall t \geq 0
$$

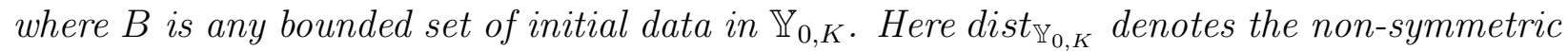
Hausdorff distance in $\mathbb{Y}_{0, K}$ and $\|B\|_{\mathbb{Y}_{0, K}}$ stands for the size of $B$ in $\mathbb{Y}_{0, K}$.

Theorem 4.2 Let $f, g \in C^{2}(\mathbb{R})$ and $\lambda \in C^{3}(\mathbb{R})$ satisfy the assumptions of Theorem 3.2. Then, $S_{K}^{s}(t)$ possesses an exponential attractor $\mathcal{M}_{K}^{s}$, bounded in $\mathbb{V}_{3} \times H^{3}(\Omega)$, namely,

$(I) \mathcal{M}_{K}^{s}$ is compact and positively invariant with respect $S_{K}^{s}(t)$, i.e.,

$$
S_{K}^{s}(t)\left(\mathcal{M}_{K}^{s}\right) \subset \mathcal{M}_{K}^{s}, \quad \forall t \geq 0
$$

(II) The fractal dimension of $\mathcal{M}_{K}^{s}$ with respect to the $\mathbb{Y}_{1, K}$-metric is finite.

(III) There exist a positive nondecreasing monotone function $Q_{s}$ and a constant $\rho_{s}>0$ such that

$$
\operatorname{dist}_{\mathbb{Y}_{1, K}}\left(S_{K}^{s}(t) B, \mathcal{M}_{K}^{s}\right) \leq Q_{s}\left(\|B\|_{\mathbb{Y}_{1, K}}\right) e^{-\rho_{s} t}, \quad \forall t \geq 0,
$$

where $B$ is any bounded set of initial data in $\mathbb{Y}_{1, K}$. Here dist $\mathbb{Y}_{1, K}$ denotes the non-symmetric Hausdorff distance in $\mathbb{Y}_{1, K}$ and $\|B\|_{\mathbb{Y}_{1, K}}$ stands for the size of $B$ in $\mathbb{Y}_{1, K}$.

The proofs are based on a fundamental result on discrete semigroups (see [14]) which is reported here below for the reader's convenience.

Theorem 4.3 Let $\mathcal{V}$ and $\mathcal{W}$ be two Banach spaces such that $\mathcal{W}$ is compactly embedded in $\mathcal{V}$. Let $X$ be a bounded subset of $\mathcal{W}$ and consider a nonlinear map $\Sigma: X \rightarrow X$ satisfying the smoothing property

$$
\left\|\Sigma\left(x_{1}\right)-\Sigma\left(x_{2}\right)\right\|_{\mathcal{W}} \leq C\left\|x_{1}-x_{2}\right\|_{\mathcal{V}},
$$

for all $x_{1}, x_{2} \in X$, where $C>0$ depends on $X$. Then the discrete dynamical system $\left(X, \Sigma^{n}\right)$ possesses a discrete exponential attractor $\mathcal{M}^{*} \subset \mathcal{V}$, that is, a compact set with finite fractal dimension such that

$$
\begin{gathered}
\Sigma\left(\mathcal{M}^{*}\right) \subset \mathcal{M}^{*}, \\
\operatorname{dist}_{\mathcal{V}}\left(\Sigma^{n}(X), \mathcal{M}^{*}\right) \leq C_{X} e^{-\rho_{*} n}, \quad n \in \mathbb{N},
\end{gathered}
$$

where $C_{X}$ and $\rho_{*}$ are positive constants independent of $n$, with the former depending on $X$. 
Remark 4.4 Theorems 4.1 and 4.2 entail that $\mathcal{A}_{K}^{w}$ and $\mathcal{A}_{K}^{s}$ have finite fractal dimension. Note that if $K=N$ then $Q_{w}, Q_{s}, \rho_{s}$ and $\rho_{w}$ depend on $M$.

The validity of the smoothing property as well as the extension of the discrete case to the continuous one are consequences of the following lemmas.

Lemma 4.5 Let the assumptions of Theorem 4.1 be satisfied. Suppose that $\mathcal{B}\left(R_{0}\right)$ is an absorbing ball of $\mathbb{Y}_{0, K}$ such that there is $t_{0}>0$ such that $S_{K}^{w}(t)\left(\mathcal{B}\left(R_{0}\right)\right) \subset \mathcal{B}\left(R_{0}\right)$ for all $t \geq t_{0}$. Set

$$
\left(\psi_{i}, \theta_{i}\right)=S_{K}^{w}(t)\left(\psi_{0 i}, \theta_{0 i}\right)
$$

where $\left(\psi_{0 i}, \theta_{0 i}\right) \in \mathcal{B}\left(R_{0}\right), i=1,2$. Then the following estimate holds

$$
\begin{aligned}
& \left\|\left(\psi_{1}-\psi_{2}\right)(t)\right\|_{\mathbb{V}_{2}}^{2}+\left\|\left(\theta_{1}-\theta_{2}\right)(t)\right\|_{\mathbb{Z}_{K}^{1}}^{2} \\
& \leq Q\left(R_{0}\right) \frac{1+\bar{t}}{\bar{t}} e^{L \bar{t}}\left(\left\|\psi_{01}-\psi_{02}\right\|_{\mathbb{V}_{1}}^{2}+\left\|\theta_{01}-\theta_{02}\right\|_{2}^{2}\right), \quad \forall \bar{t}>0,
\end{aligned}
$$

where $\bar{t}:=t-t_{0}$. Here $L$ is a positive constant and $Q$ is a monotone increasing function which only depend on $R_{0}, \Omega, \Gamma$ and on the structural parameters of the problem.

Proof. Let us consider (2.49)-(2.50) and recall that there we have set $\psi=\psi_{1}-\psi_{2}$ and $\theta=\theta_{1}-\theta_{2}$. We proceed as in [21] by differentiating (2.49) with respect to $t$ and then taking $u=\psi_{t}(t)$, adding the resulting relationship to (2.50) where we take $v=\theta_{t}(t)$. Hence we have

$$
\begin{aligned}
& \frac{d}{d t}\left[\left\|\psi_{t}(t)\right\|_{\mathbb{V}_{0}}^{2}+\|\nabla \theta(t)\|_{2}^{2}+d\left\|\theta_{\mid \Gamma}(t)\right\|_{2, \Gamma}^{2}\right]+2\left\|\psi_{t}(t)\right\|_{\mathbb{V}_{1}}^{2}+2\left\|\theta_{t}(t)\right\|_{2}^{2} \\
& =-2\left\langle\partial_{t}\left(\Upsilon_{1}(t)\right), \psi_{t}(t)\right\rangle_{2}+2\left\langle\partial_{t}\left(\Upsilon_{2}(t)\right), \psi_{t}(t)\right\rangle_{2, \Gamma} \\
& +2\left\langle\partial_{t}\left(\Upsilon_{3}(t)\right), \psi_{t}(t)\right\rangle_{2}-2\left\langle\Upsilon_{4}(t), \theta_{t}(t)\right\rangle_{2} .
\end{aligned}
$$

We recall that $\Upsilon_{1}, \Upsilon_{2}$ and $\Upsilon_{3}$ are defined by (2.51)-(2.52). Moreover, we have

$$
\partial_{t} \Upsilon_{1}=f^{\prime}\left(\psi_{1}\right) \partial_{t} \psi_{1}-f^{\prime}\left(\psi_{2}\right) \partial_{t} \psi_{2}=f^{\prime}\left(\psi_{1}\right) \partial_{t} \psi+\left[f^{\prime}\left(\psi_{1}\right)-f^{\prime}\left(\psi_{2}\right)\right] \partial_{t} \psi_{2},
$$

where a similar formula holds for $\partial_{t} \Upsilon_{2}$. Moreover, it is easy to check that

$$
\begin{aligned}
\partial_{t} \Upsilon_{3} & =\lambda^{\prime \prime}\left(\psi_{1}\right) \psi_{t} \theta_{1}+\lambda^{\prime \prime}\left(\psi_{1}\right) \psi_{2 t} \theta+\left[\lambda^{\prime \prime}\left(\psi_{1}\right)-\lambda^{\prime \prime}\left(\psi_{2}\right)\right] \psi_{2 t} \theta_{2} \\
& +\lambda^{\prime}\left(\psi_{1}\right) \theta_{t}+\left[\lambda^{\prime}\left(\psi_{1}\right)-\lambda^{\prime}\left(\psi_{1}\right)\right] \theta_{2 t} .
\end{aligned}
$$

Consequently, from (3.25) and the (local) Lipschitz continuity of $f^{\prime}$ and $g^{\prime}$ it follows that

$$
\left\|\partial_{t}\left(\Upsilon_{1}(t)\right)\right\|_{2}+\left\|\partial_{t}\left(\Upsilon_{2}(t)\right)\right\|_{2, \Gamma} \leq Q\left(R_{0}\right)\left(\left\|\psi_{t}(t)\right\|_{\mathbb{V}_{0}}+\left\|\psi_{2 t}(t)\right\|_{\mathbb{V}_{1}}\|\psi(t)\|_{\mathbb{V}_{1}}\right),
$$


for all $t \geq t_{0}$. Here we have also used the embedding $H^{1}(\Omega) \hookrightarrow L^{4}(\Omega)$. Moreover, owing to (3.25), (3.38)-(3.41), the (local) Lipschitz continuity of $\lambda^{\prime \prime}$ also implies that

$$
\begin{aligned}
& 2\left|\left\langle\partial_{t}\left(\Upsilon_{3}(t)\right), \psi_{t}(t)\right\rangle_{2}-\left\langle\Upsilon_{4}(t), \theta_{t}(t)\right\rangle_{2}\right| \\
& \leq Q\left(R_{0}\right)\left\|\psi_{2 t}(t)\right\|_{H^{1}(\Omega)}^{2}\left(\|\psi(t)\|_{H^{1}(\Omega)}^{2}+\|\theta(t)\|_{2}^{2}\right) \\
& +Q\left(R_{0}\right)\left\|\psi_{t}(t)\right\|_{2}^{2}+C\left\|\theta_{2 t}\right\|_{H^{1}(\Omega)}^{2}\|\psi(t)\|_{H^{1}(\Omega)}^{2} \\
& +\left\|\theta_{t}(t)\right\|_{2}^{2}+\left\|\psi_{t}(t)\right\|_{H^{1}(\Omega)}^{2},
\end{aligned}
$$

for all $t \geq t_{0}$. Consequently, owing to (4.8)-(4.10) and (3.38)-(3.40), from (4.7) we infer

$$
\begin{aligned}
& \frac{d}{d t}\left[\left\|\psi_{t}(t)\right\|_{\mathbb{V}_{0}}^{2}+\|\nabla \theta(t)\|_{2}^{2}+d\left\|\theta_{\mid \Gamma}(t)\right\|_{2, \Gamma}^{2}\right]+\left\|\psi_{t}(t)\right\|_{\mathbb{V}_{1}}^{2}+\left\|\theta_{t}(t)\right\|_{2}^{2} \\
& \leq Q\left(R_{0}\right)\left(\|\psi(t)\|_{H^{1}(\Omega)}^{2}+\|\theta(t)\|_{2}^{2}+\left\|\psi_{t}(t)\right\|_{2}^{2}\right)
\end{aligned}
$$

for all $t \geq t_{0}$. Multiply now inequality (4.11) by $\bar{t}:=t-t_{0}$ and integrate over $\left(t_{0}, t\right)$ the resulting relation. Then we deduce that

$$
\begin{aligned}
& \bar{t} Y_{2}(t)+\int_{t_{0}}^{t} \bar{s}\left(\left\|\psi_{t}(s)\right\|_{\mathbb{V}_{1}}^{2}+\left\|\theta_{t}(s)\right\|_{2}^{2}\right) d s \\
& \leq Q\left(R_{0}\right) \int_{t_{0}}^{t} \bar{s}\left(\|\psi(s)\|_{H^{1}(\Omega)}^{2}+\|\theta(s)\|_{2}^{2}+\left\|\psi_{t}(s)\right\|_{2}^{2}\right) d s+\int_{t_{0}}^{t} Y_{2}(s) d s
\end{aligned}
$$

for all $t \geq t_{0}$, where $\bar{s}:=s-t_{0}$ and $Y_{2}$ is defined as

$$
Y_{2}(t):=\left\|\psi_{t}(t)\right\|_{\mathbb{V}_{0}}^{2}+\|\nabla \theta(t)\|_{2}^{2}+d\left\|\theta_{\mid \Gamma}(t)\right\|_{2, \Gamma}^{2} .
$$

Finally, exploiting inequality (2.48) once more to estimate the right-hand side of (4.12), it is not difficult to show

$$
\begin{aligned}
& \bar{t} Y_{2}(t)+\int_{t_{\#}}^{t} \bar{s}\left(\left\|\psi_{t}(s)\right\|_{\mathbb{V}_{1}}^{2}+\left\|\theta_{t}(s)\right\|_{2}^{2}\right) d s \\
& \leq Q\left(R_{0}\right)(1+\bar{t}) e^{L_{w} t}\left(\left\|\psi_{01}-\psi_{02}\right\|_{\mathbb{V}_{1}}^{2}+\left\|\theta_{01}-\theta_{02}\right\|_{2}^{2}\right), \quad \forall t \geq t_{0},
\end{aligned}
$$

which yields the following estimate

$$
\begin{aligned}
& \left\|\psi_{t}(t)\right\|_{\mathbb{V}_{0}}^{2}+\|\theta(t)\|_{\mathbb{Z}_{K}^{1}}^{2}+\frac{1}{\bar{t}} \int_{t_{\#}}^{t} \bar{s}\left[\left\|\psi_{t}(s)\right\|_{\mathbb{V}_{1}}^{2}+\left\|\theta_{t}(s)\right\|_{2}^{2}\right] d s \\
& \leq Q\left(R_{0}\right) \frac{1+\bar{t}}{\bar{t}} e^{L_{w} \bar{t}}\left(\left\|\psi_{01}-\psi_{02}\right\|_{\mathbb{V}_{1}}^{2}+\left\|\theta_{01}-\theta_{02}\right\|_{2}^{2}\right), \quad \forall t>t_{0} .
\end{aligned}
$$


Here we have also used (2.48). On account of (4.13), we can argue exactly as in the proof of Lemma 3.4 (see (3.33)) to get the required estimate on the $\mathbb{V}_{2}$-norm of $\psi(t)$. The desired inequality (4.6) then follows immediately from (4.13). We leave the rigorous details to the reader.

The following lemma is a straightforward consequence of Lemma 4.5.

Lemma 4.6 Let the assumptions of Theorem 4.2 be satisfied. Suppose that $\mathcal{B}\left(R_{1}\right)$ is an absorbing ball of $\mathbb{Y}_{1, K}$ such that there is $t_{1}>0$ such that $S_{K}^{s}(t)\left(\mathcal{B}\left(R_{1}\right)\right) \subset \mathcal{B}\left(R_{1}\right)$ for all $t \geq t_{1}$. Set

$$
\left(\psi_{i}, \theta_{i}\right)=S_{K}^{s}(t)\left(\psi_{0 i}, \theta_{0 i}\right),
$$

where $\left(\psi_{0 i}, \theta_{0 i}\right) \in \mathcal{B}\left(R_{1}\right), i=1,2$. Then the function $\left(\psi_{1}-\psi_{2}, \theta_{1}-\theta_{2}\right)$ satisfies the same estimate of (4.6), with $\bar{t}:=t-t_{1}$, and $L, Q$ depending on $R_{1}, \Omega, \Gamma$ and on the structural parameters of the problem.

The following lemmas are concerned with the time regularity of $S_{K}^{w}(t)$ and $S_{K}^{s}(t)$, respectively.

Lemma 4.7 Let the assumptions of Lemma 3.4 hold. Then, there is a positive monotone increasing function $Q$ such that

$$
\left\|S_{K}^{w}(t)\left(\psi_{0}, \theta_{0}\right)-S_{K}^{w}(\tilde{t})\left(\psi_{0}, \theta_{0}\right)\right\|_{\mathbb{Y}_{0, K}} \leq Q\left(R_{0}\right)|t-\tilde{t}|^{1 / 2}
$$

for all $t, \tilde{t} \in\left[t_{0},+\infty\right)$ and any $\left(\psi_{0}, \theta_{0}\right) \in \mathcal{B}\left(R_{0}\right)$.

Proof. From (3.27) we deduce

$$
\int_{t_{0}}^{+\infty}\left\|\partial_{s} S_{K}^{j}(s)\left(\psi_{0}, \theta_{0}\right)\right\|_{\mathbb{V}_{1} \times L^{2}(\Omega)}^{2} d s \leq Q\left(R_{0}\right)
$$

Observing that

$$
S_{K}^{j}(t)\left(\psi_{0}, \theta_{0}\right)-S_{K}^{j}(\tilde{t})\left(\psi_{0}, \theta_{0}\right)=\int_{t}^{\tilde{t}} \partial_{s} S_{K}^{w}(s)\left(\psi_{0}, \theta_{0}\right) d s
$$

thanks to (4.15), we deduce (4.14).

Lemma 4.8 Let the assumptions of Lemma 3.5 hold. Then, there is a positive monotone increasing function $Q$ such that

$$
\left\|S_{K}^{s}(t)\left(\psi_{0}, \theta_{0}\right)-S_{K}^{s}(\tilde{t})\left(\psi_{0}, \theta_{0}\right)\right\|_{\mathbb{Y}_{1, K}} \leq Q\left(R_{1}\right)\left(|t-\tilde{t}|^{1 / 6}+|t-\tilde{t}|^{1 / 4}\right),
$$

for all $t, \tilde{t} \in\left[t_{1},+\infty\right)$ and any $\left(\psi_{0}, \theta_{0}\right) \in \mathcal{B}\left(R_{1}\right)$. 
Proof. We still have (4.15). Moreover, from (3.34) holds. Thus, using standard interpolation inequalities and Hölder's inequality, from (4.16) we deduce

$$
\begin{aligned}
& \left\|S_{K}^{s}(t)\left(\psi_{0}, \theta_{0}\right)-S_{K}^{s}(\tilde{t})\left(\psi_{0}, \theta_{0}\right)\right\|_{\mathbb{Y}_{1, K}}^{2} \\
& =\|\psi(t)-\psi(\tilde{t})\|_{\mathbb{V}_{2}}^{2}+\|\theta(t)-\theta(\tilde{t})\|_{\mathbb{Z}_{K}^{1}}^{2} \\
& \leq Q\left(R_{0}\right)\left(\|\psi(t)-\psi(\tilde{t})\|_{\mathbb{V}_{0}}^{2 / 3}+\|\theta(t)-\theta(\tilde{t})\|_{2}\right) \\
& \leq Q\left(R_{0}\right)\left(|t-\tilde{t}|^{1 / 3}+|t-\tilde{t}|^{1 / 2}\right),
\end{aligned}
$$

for all $t>t_{1}$, that is, (4.17).

Proof of Theorem 4.1. Using Lemma 3.3, Lemma 3.4 and Lemma 4.5, we can find a bounded subset $X$ of $\mathbb{Y}_{0, K} \cap\left(\mathbb{V}_{2} \times H^{2}(\Omega)\right)$ and $t^{*}>0$ such that, setting $\Sigma_{K}=S_{K}^{w}\left(t^{*}\right)$, the mapping $\Sigma_{K}: X \rightarrow X$ enjoys the smoothing property (4.6). Therefore Theorem 4.3 applies to $\Sigma_{K}$ and there exists a compact set $\mathcal{M}_{K}^{w, *} \in X$ of finite fractal dimension (with respect to $\mathbb{Y}_{0, K}$-metric) that satisfies (4.4) and (4.5). Hence, setting

$$
\mathcal{M}_{K}^{w}=\cup_{t \in\left[t^{*}, 2 t^{*}\right]} S_{K}^{w}(t) \mathcal{M}_{K}^{w, *}
$$

we have that $(I)$ and $(I I I)$ of Theorem 4.1 are fulfilled, while $(I I)$ is a consequence of (2.48) and (4.14).

Proof of Theorem 4.2. Set now $\mathcal{V}:=\mathbb{V}_{1} \times L^{2}(\Omega)$ and $\mathcal{W}:=\mathbb{V}_{2} \times \mathbb{Z}_{K}^{1}$ in Theorem 4.3. Using Lemma 2.5, Lemma 3.5 and Lemma 4.6, we can find a bounded subset $X$ of $\mathbb{Y}_{1, K} \cap\left(\mathbb{V}_{3} \times H^{3}(\Omega)\right)$ and $t^{+}>0$ such that, setting $\Sigma_{K}=S_{K}^{s}\left(t^{+}\right)$, the mapping $\Sigma_{K}: X \rightarrow X$ enjoys the smoothing property (4.6). Therefore Theorem 4.3 applies to $\Sigma_{K}$ and there exists a compact set $\mathcal{M}_{K}^{s, *} \in X$ of finite fractal dimension (with respect to $\mathbb{Y}_{0, K}$-metric) that satisfies (4.4) and (4.5). Hence, setting as above,

$$
\mathcal{M}_{K}^{s}=\cup_{t \in\left[t^{+}, 2 t^{+}\right]} S_{K}^{s}(t) \mathcal{M}_{K}^{s, *},
$$

we have that $(I),(I I)$ and $(I I I)$ of Theorem 4.2 are fulfilled, but with the metric of $\mathbb{Y}_{1, K}$ being replaced by that of $\mathbb{Y}_{0, K}$. More precisely, $\mathcal{M}_{K}^{s}$ has finite fractal dimension (with respect to the metric of $\mathbb{Y}_{0, K}$ ), whereas only the following inequality is valid:

$$
\operatorname{dist}_{\mathbb{Y}_{0, K}}\left(S_{K}^{s}(t) B, \mathcal{M}_{K}^{s}\right) \leq Q_{s}\left(\|B\|_{\mathbb{Y}_{1, K}}\right) e^{-\rho_{s} t}, \quad \forall t \geq 0 .
$$

In order to obtain the finite-dimensionality and the required exponential convergence (4.2) of $\mathcal{M}_{K}^{s}$ in the required metric of $\mathbb{Y}_{1, K}$, it remains to recall that $S_{K}^{s}(t), t \geq t_{1}$, possesses the smoothing property (3.34) and to use the standard interpolation inequalities $\|\cdot\|_{\mathbb{V}_{2}} \leq$ $C\|\cdot\|_{\mathbb{V}_{0}}^{1 / 3}\|\cdot\|_{\mathbb{V}_{3}}^{2 / 3},\|\cdot\|_{\mathbb{Z}_{K}^{1}} \leq C\|\cdot\|_{2}^{1 / 2}\|\cdot\|_{H^{2}}^{1 / 2}$, where the constant $C$ is independent of $t$. The proof of Theorem 4.2 is now complete. 


\section{Convergence to single equilibria}

Here we work within the framework used in [20]. Therefore, we do not make any growth assumption on $f$ and $g$ and we consider solutions given by Theorem ??. However, Theorem 5.8 below also holds for solutions considered in the previous sections, thanks to the smoothing effects (see Lemma 3.4).

Let us begin by recalling the following proposition (see [20, Remark 15]).

Proposition 5.1 Fix $K \in\left\{D, N, R, W_{0}, W_{1}\right\}$, and let the hypotheses of Theorem ?? hold. The semigroup $\mathcal{S}_{K}^{\alpha}(t)$ has a (strict) Lyapunov functional defined by the free energy, namely,

$$
\begin{gathered}
\mathcal{L}_{K}\left(\psi_{0}, \theta_{0}\right)=\frac{1}{2}\left[\left\|\nabla \psi_{0}\right\|_{2}^{2}+\alpha\left\|\nabla_{\Gamma} \psi_{0}\right\|_{2, \Gamma}^{2}+\beta\left\|\psi_{0}\right\|_{2, \Gamma}^{2}+\left\|\theta_{0}\right\|_{2}^{2}+\frac{a}{b}\left\|\theta_{0}\right\|_{2, \Gamma}^{2}\right] \\
+\int_{\Omega} F\left(\psi_{0}\right) d x+\int_{\Gamma} G\left(\psi_{0}\right) d S,
\end{gathered}
$$

where $F$ and $G$ are primitives of $f$ and $g$, respectively. In particular, we have, for all $t>0$,

$$
\frac{d}{d t} \mathcal{L}_{K}\left(\mathcal{S}_{K}^{\alpha}(t)\left(\psi_{0}, \theta_{0}\right)\right)=-\left\|\psi_{t}(t)\right\|_{2}^{2}-\left\|\psi_{t}(t)\right\|_{2, \Gamma}^{2}-\|\nabla \theta(t)\|_{2}^{2}-\frac{c}{b}\left\|\theta_{\mid \Gamma}(t)\right\|_{2, \Gamma}^{2} .
$$

We also have (cf. [20, Lemma 11])

Proposition 5.2 Let $\alpha>0$ and suppose that $f, g \in C^{1}(\mathbb{R})$ satisfying assumptions (??) and (??) when $K \in\left\{N, W_{0}\right\}$. Then, for each $K \in\left\{D, N, R, W_{0}, W_{1}\right\}$, the semigroup $\mathcal{S}_{K}^{\alpha}(t)$ acting on the complete metric space $\mathbb{W}_{\alpha, K}:=\left(\mathbb{V}_{\alpha}^{2} \times \mathbb{Z}_{K}^{1}\right) \cap \mathbb{Y}_{\alpha, a}$ has a bounded absorbing set.

Let us now examine more closely the set of equilibria. We observe first that $\left(\psi_{\infty}, \theta_{\infty}\right) \in$ $\mathbb{W}_{\alpha, K}$ is an equilibrium for $\mathbf{P}_{K}^{\alpha}$ if and only if it is a solution to the boundary value problem

$$
\begin{gathered}
-\Delta \psi_{\infty}+f\left(\psi_{\infty}\right)-\lambda_{0} \theta_{\infty}=0, \quad \text { in } \Omega, \\
-\alpha \Delta_{\Gamma} \psi_{\infty}+\partial_{\mathbf{n}} \psi_{\infty}+\beta \psi_{\infty}+g\left(\psi_{\infty}\right)=0, \\
-\Delta \theta_{\infty}=0, \quad \text { in } \Omega, \\
b \partial_{\mathbf{n}} \theta_{\infty}+c \theta_{\infty}=0, \quad \text { on } \Gamma .
\end{gathered}
$$

Then, it is not difficult to realize that when $K \in\left\{D, R, W_{1}\right\}$, then $\theta_{\infty} \equiv 0$. Otherwise, when $K \in\left\{N, W_{0}\right\}, \theta_{\infty}$ must be equal to a constant which is uniquely determined from (3.14) or (3.15). Thus, the above stationary problem reduces to the following

$$
\left\{\begin{aligned}
-\Delta \psi_{\infty}+f\left(\psi_{\infty}\right)-\lambda_{0} \theta_{\infty}=0, & \text { in } \Omega, \\
-\alpha \Delta_{\Gamma} \psi_{\infty}+\partial_{\mathbf{n}} \psi_{\infty}+\beta \psi_{\infty}+g\left(\psi_{\infty}\right)=0, & \text { on } \Gamma
\end{aligned}\right.
$$

where

$$
\theta_{\infty}=\left\{\begin{array}{c}
0, \quad \text { for } K \in\left\{D, R, W_{1}\right\}, \\
I_{K}\left(\psi_{0}, \theta_{0}\right)-\frac{\lambda_{0}|\Omega|}{|\Omega|+\frac{a}{b}|\Gamma|}\left\langle\psi_{\infty}\right\rangle_{\Omega}, \quad \text { for } K \in\left\{N, W_{0}\right\},
\end{array}\right.
$$


and $\langle\cdot\rangle_{\Omega}$ stands for the spatial average over $\Omega$. It is not difficult to check that (5.3) possesses at least one solution (i.e., there exists at least one stationary solution) by means of standard arguments (see, e.g., [7, 10, 48]).

We now recall a further result that can be inferred from [20, Lemma 13 and Remark 14] which yields the relative compactness in $\mathbb{W}_{\alpha, K}$ of any trajectory.

Lemma 5.3 Let the assumptions of Proposition 5.2 hold. For any initial datum $\left(\psi_{0}, \theta_{0}\right) \in$ $\mathbb{W}_{\alpha, K}$, the corresponding solution to $\mathbf{P}_{K}^{\alpha}$ satisfies the following estimates, namely, for any $\epsilon>0$ there exists $C_{\epsilon}>0$ such that

$$
\|(\psi(t), \theta(t))\|_{\mathbb{V}_{\alpha}^{3} \times \mathbb{Z}_{K}^{l}}+\left\|\psi_{t}(t)\right\|_{\mathbb{V}_{\alpha}^{1}}+\left\|\theta_{t}(t)\right\|_{\mathbb{Z}_{K}^{1}} \leq C_{\epsilon}, \quad t \geq \epsilon>0
$$

where $C_{\epsilon}>0$ depends only on $\epsilon$ and on the norm of the initial datum in $\mathbb{V}_{\alpha}^{2} \times \mathbb{Z}_{K}^{1}$. Here, $l=2$ if $K \in\left\{W_{0}, W_{1}\right\}$ and $l=3$ otherwise.

Remark 5.4 We recall that in [20] we proved the existence of a compact absorbing set (compare with Lemma 3.4). Therefore, in the hypotheses of Proposition 5.2, the semigroup $\mathcal{S}_{K}^{\alpha}(t)$ has a connected global attractor $\mathcal{A}^{K}$ which, due to Proposition 5.1, coincides with the unstable manifold of the set $\mathcal{P}_{K}$ of the stationary points (cf., e.g., [47, Chap.7, Sec.4]).

Therefore, on account of the above results, we report some standard implications.

Lemma 5.5 For any $\left(\psi_{0}, \theta_{0}\right) \in \mathbb{W}_{\alpha, K}$, the set $\omega\left(\psi_{0}, \theta_{0}\right)$ is a nonempty compact connected subset of $\mathbb{W}_{\alpha, K}$. Furthermore, we have:

(i) $\omega\left(\psi_{0}, \theta_{0}\right)$ is fully invariant for $\mathcal{S}_{K}^{\alpha}(t)$;

(ii) $\mathcal{L}_{K}$ is constant on $\omega\left(\psi_{0}, \theta_{0}\right)$;

(iii) $\operatorname{dist}_{\mathbb{W}_{\alpha, K}}\left(\mathcal{S}_{K}^{\alpha}(t)\left(\psi_{0}, \theta_{0}\right), \omega\left(\psi_{0}, \theta_{0}\right)\right) \rightarrow 0$ as $t \rightarrow+\infty$;

(iv) $\omega\left(\psi_{0}, \theta_{0}\right)$ consists of equilibria only.

Next, following an argument in [7], without loss of generality, we assume that $\left(\psi_{0}, \theta_{0}\right)$ satisfies the condition $I_{K}\left(\psi_{0}, \theta_{0}\right)=0$, whenever $K \in\left\{N, W_{0}\right\}$. Indeed it suffices to replace the solution $(\psi, \theta)$ corresponding to $\left(\psi_{0}, \theta_{0}\right)$ by $(\psi-c, \theta)$ with $c=\left(|\Omega|+\frac{a}{b}|\Gamma|\right) I_{K}(\psi, \theta) /\left(|\Omega| \lambda_{0}\right)$ and to note that $(\psi-c, \theta)$ satisfies the phase-field equations (??)-(1.3) with $f(y)$ and $g(y)$ replaced by $f(y+c)$ and $g(y+c)$, respectively. The convergence of $(\psi(t), \theta(t))$ converges as $t \rightarrow+\infty$ is not affected by this normalization. Thus, recalling (3.18), from now on we will consider $\mathbb{Y}_{\alpha, a}$ with $M=0$ if $K \in\left\{N, W_{0}\right\}$. Therefore $\mathbb{Y}_{\alpha, a}$ is a Hilbert space and we will indicate by $\mathbb{Y}_{\alpha, a}^{*}$ its dual. Moreover, if $K \in\left\{N, W_{0}\right\}$, in the functional $\mathcal{L}_{K}: \mathbb{Y}_{\alpha, a} \rightarrow \mathbb{R}$ we will replace $F(y)$ and $G(y)$ by $F(y+c)$ and $G(y+c)$, respectively.

The version of the Łojasiewicz-Simon inequality we need is given by

Lemma 5.6 Let $\left(\psi_{\infty}, \theta_{\infty}\right) \in \mathbb{W}_{\alpha, K}$ satisfy (5.3)-(2.36), that is, $\left(\psi_{\infty}, \theta_{\infty}\right)$ is a critical point of $\mathcal{L}_{K}$. Assume that $F$ and $G$ are real analytic. There exist constants $\xi \in(0,1 / 2)$ and $C_{L}>0, \zeta>0$ depending on $\left(\psi_{\infty}, \theta_{\infty}\right)$ such that, for any $(\psi, \theta) \in \mathbb{W}_{\alpha, K}$, if

$$
\left\|(\psi, \theta)-\left(\psi_{\infty}, \theta_{\infty}\right)\right\|_{\mathbb{V}_{\alpha}^{2} \times \mathbb{Z}_{K}^{1}} \leq \zeta
$$


denoting by $\mathcal{L}_{K}^{\prime}$ the Fréchet derivative of $\mathcal{L}_{K}$, we have

$$
C_{L}\left\|\mathcal{L}_{K}^{\prime}(\psi, \theta)\right\|_{\mathbb{Y}_{\alpha, a}^{*}} \geq\left|\mathcal{L}_{K}(\psi, \theta)-\mathcal{L}_{K}\left(\psi_{\infty}, \theta_{\infty}\right)\right|^{1-\xi}
$$

Remark 5.7 The proof of Lemma 5.6 is based on the same arguments used in [7] (see also [10]). Indeed, $\psi$ is bounded so that the nonlinearities $f(\psi), g(\psi)$ as well as their higher-order derivatives are bounded too.

We can now state the main result of this section.

Theorem 5.8 Fix $K \in\left\{D, N, R, W_{0}, W_{1}\right\}$. Let the assumptions of Proposition 5.2 hold and suppose, in addition, that the nonlinearities $F$ and $G$ are real analytic. For any given initial datum $\left(\psi_{0}, \theta_{0}\right) \in \mathbb{W}_{\alpha, K}$, the solution $(\psi(t), \theta(t))=\mathcal{S}_{K}^{\alpha}(t)\left(\psi_{0}, \theta_{0}\right)$ to $\mathbf{P}_{K}^{\alpha}$ converges to a single equilibrium $\left(\psi_{\infty}, \theta_{\infty}\right)$ in the topology of $\mathbb{W}_{\alpha, K}$, that is,

$$
\lim _{t \rightarrow+\infty}\left(\left\|\psi(t)-\psi_{\infty}\right\|_{\mathbb{V}_{\alpha}^{2}}+\left\|\theta(t)-\theta_{\infty}\right\|_{\mathbb{Z}_{K}^{1}}\right)=0
$$

Moreover, there exist $C \geq 0$ and $\xi \in(0,1 / 2)$ depending on $\left(\psi_{\infty}, \theta_{\infty}\right)$ such that

$$
\left\|\psi(t)-\psi_{\infty}\right\|_{\mathbb{V}_{\alpha}^{2}}+\left\|\theta(t)-\theta_{\infty}\right\|_{\mathbb{Z}_{K}^{1}}+\left\|\psi_{t}(t)\right\|_{\mathbb{V}_{\alpha}^{0}} \leq C(1+t)^{-\xi /(1-2 \xi)},
$$

for all $t \geq 0$.

\section{References}

[1] S.M. Allen, J.W. Cahn, A microscopic theory for the antiphase boundary motion and its application to antiphase domain coarsening, Acta Metallurgica 27 (1979), 1085-1095.

[2] P.W. Bates, S. Zheng, Inertial manifolds and inertial sets for the phase-field equations, J. Dynamics Differential Equations 4 (1992), 375-397.

[3] D. Brochet, X. Chen, D. Hilhorst, Finite dimensional exponential attractor for the phase-field model, Appl. Anal. 49 (1993), 197-212.

[4] D. Brochet, D. Hilhorst, Universal attractor and inertial sets for the phase-field model, Appl. Math. Lett. 4 (1991), 59-62.

[5] M. Brokate, J. Sprekels, Hysteresis and Phase Transitions, Springer, New York, 1996.

[6] G. Caginalp, An analysis of a phase field model of a free boundary, Arch. Ration. Mech. Anal. 92 (1986), 205-245.

[7] R. Chill, E. Fašangová, J. Prüss, Convergence to steady states of solutions of the CahnHilliard and Caginalp equations with dynamic boundary conditions, Math. Nachr. 13 (2006), 1448-1462. 
[8] L. Cherfils, S. Gatti, A. Miranville, Existence of global solutions to the Caginalp phasefield system with dynamic boundary conditions and singular potentials, J. Math. Anal. Appl. 343 (2008), 557-566.

[9] L. Cherfils, A. Miranville, Some remarks on the asymptotic behavior of the Caginalp system with singular potentials, Adv. Math. Sci. Appl. 16 (2007), 107-129.

[10] L. Cherfils, A. Miranville, On the Caginalp system with dynamic boundary conditions and singular potentials, Appl. Math., to appear.

[11] J.B. Collins, H. Levine, Diffuse interface models of diffusion limited crystal growth, Phys. Rev. B 31 (1985), 6119-6222 [Errata, Phys. Rev. B 33 (1985), 2020].

[12] A. Damlamian, N. Kenmochi, N. Sato, Subdifferential operator approach to a class of nonlinear systems for Stefan problems with phase relaxation, Nonlinear Anal. 23 (1994), 115-142.

[13] E. Hebey, Nonlinear Analysis on Manifolds: Sobolev Spaces and Inequalities, Courant Lecture Notes in Mathematics 5, American Mathematical Society, Providence, 1999.

[14] M. Efendiev, A. Miranville, S. Zelik, Exponential attractors for a nonlinear reactiondiffusion system in $\mathbb{R}^{3}$, C. R. Math. Acad. Sci. Paris 330 (2000), 713-718.

[15] C.M. Elliott, S. Zheng, Global existence and stability of solutions to the phase-field equations, in "Free boundary problems", Internat. Ser. Numer. Math. 95, 46-58, Birkhäuser Verlag, Basel, 1990.

[16] H.P. Fischer, Ph. Maass, W. Dieterich, Novel surface modes of spinodal decomposition, Phys. Rev. Letters 79 (1997), 893-896.

[17] H.P. Fischer, Ph. Maass, W. Dieterich, Diverging time and length scales of spinodal decomposition modes in thin flows, Europhys. Letters 62 (1998), 49-54.

[18] C.G. Gal, Well-posedness and long time behavior of the non-isothermal viscous CahnHilliard model with dynamic boundary conditions, Dyn. Partial Differ. Equ. 5 (2008), 39-67.

[19] C.G. Gal, G.R. Goldstein, J.A. Goldstein, S. Romanelli, Fredholm alternative, nonlinear elliptic problems with Wentzell boundary conditions, Calc. Var. Partial Differential Equations, to appear.

[20] C.G. Gal, M. Grasselli, The nonisothermal Allen-Cahn equation with dynamic boundary conditions, Discrete Contin. Dyn. Syst., 22 (2008), 1009-1040.

[21] C.G. Gal, M. Grasselli, On the asymptotic behavior of the Caginalp system with dynamic boundary conditions, Commun. Pure Appl. Anal. 8 (2009), 689-710. 
[22] C.G. Gal, M. Grasselli, A. Miranville, Robust exponential attractors for phase-field equations with dynamic boundary conditions, NoDEA Nonlinear Differential Equations Appl., 15 (2008), 535-556.

[23] C.G. Gal, M. Grasselli, A. Miranville, Nonisothermal Allen-Cahn equations with coupled dynamic boundary conditions, in "Nonlinear phenomena with energy dissipation: mathematical analysis, modeling and simulation "(P. Colli, A. Damlamian, N. Kenmochi, M. Mimura, J. Sprekels, Eds.), Gakuto Int. Ser. Math. Sci. Appl. 29, 117-139, Gakkōtosho, Tokyo 2008.

[24] S. Gatti, A. Miranville, Asymptotic behavior of phase-field system with dynamic boundary conditions, in "Differential Equations: Inverse and Direct Problems"(A. Favini, A. Lorenzi, Eds.), Ser. Lect. Notes Pure Appl. Math. 251, 149-170, Chapman \& Hall/CRC, Boca Raton, 2006.

[25] C. Giorgi, M. Grasselli, V. Pata, Uniform attractors for a phase-field model with memory and quadratic nonlinearity, Indiana Univ. Math. J. 48 (1999), 1395-1445.

[26] M. Grasselli, V. Pata, Asymptotic behavior of a parabolic-hyperbolic system, Commun. Pure Appl. Anal. 3 (2004), 849-881.

[27] M. Grasselli, H. Petzeltová, G. Schimperna, Long time behavior of solutions to the Caginalp system with singular potential, Z. Anal. Anwendungen 25 (2006), 51-72.

[28] M. Grasselli, H. Wu, S. Zheng, Asymptotic behavior of a non-isothermal GinzburgLandau model, Quart. Appl. Math. 66 (2008), 743-770.

[29] A. Haraux, Systèmes dynamiques dissipatifs et applications, Masson, Paris, 1991.

[30] A. Jiménez-Casas, A. Rodríguez-Bernal, Asymptotic behaviour for a phase field model in higher order Sobolev spaces, Rev. Mat. Complut. 15 (2002), 213-248.

[31] V.K. Kalantarov, On the minimal global attractor of a system of phase field equations (Russian), Zap. Nauchn. Sem. Leningrad. Otdel. Mat. Inst. Steklov. (LOMI) 188 (1991), Kraev. Zadachi Mat. Fiz. i Smezh. Voprosy Teor. Funktsii. 22, 70-86, 186 [translation in J. Math. Sci. 70 (1994), no. 3, 1767-1777].

[32] O.V. Kapustyan, An attractor of a semiflow generated by a system of phase-field equations without uniqueness of the solution (Ukrainian), Ukraïn. Mat. Zh. 51 (1999), 10061009 [Translation in Ukrainian Math. J. 51 (1999), no.7, 1135-1139 (2000)].

[33] R. Kenzler, F. Eurich, Ph. Maass, B. Rinn, J. Schropp, E. Bohl, W. Dieterich, Phase separation in confined geometries: Solving the Cahn-Hilliard equation with generic boundary conditions, Computer Phys. Comm. 133 (2001), 139-157.

[34] $\mathrm{Ph}$. Laurençot, Long-time behaviour for a model of phase-field type, Proc. Roy. Soc. Edinburgh Sect.A 126 (1996), 167-185. 
[35] S. Łojasiewicz, Une propriété topologique des sous-ensembles analytiques réels, Colloque Internationaux du C.N.R.S. 117, Les Équations aux Dérivées Partielles (Paris, 1962) pp. 87-89, Éditions du Centre National de la Recherche Scientifique, Paris, 1963.

[36] S. Łojasiewicz, Sur la geometrie semi- et sous-analytique, Ann. Inst. Fourier (Grenoble) 43 (1993), 1575-1595.

[37] H. Matano, Convergence of solutions of one-dimensional semilinear heat equation, J. Math. Kyoto Univ. 18 (1978), 221-227.

[38] G.B. McFadden, Phase-field models of solidification, Contemp. Math. 306 (2002), 107145.

[39] A. Miranville, S. Zelik, Exponential attractors for the Cahn-Hilliard equation with dynamical boundary conditions, Math. Models Appl. Sci. 28 (2005), 709-735.

[40] J. Prüss, R. Racke, S. Zheng, Maximal regularity and asymptotic behavior of solutions for the Cahn Hilliard equation with dynamic boundary conditions, Ann. Mat. Pura Appl. (4) 185 (2006), 627-648.

[41] P. Polačik, F. Simondon, Nonconvergent bounded solutions of semilinear heat equations on arbitrary domains, J. Differential Equations 186 (2002), 586-610.

[42] V. Pata, S. Zelik, A result on the existence of global attractors for semigroups of closed operators, Commun. Pure Appl. Anal. 6 (2007), 481-486.

[43] J. Prüss, M. Wilke, Maximal $\mathrm{L}^{p}$ regularity for the Cahn Hilliard equation with nonconstant temperature and dynamic boundary conditions, in "Partial differential equations and functional analysis", Oper. Theory Adv. Appl. 168, 209-236, Birkhäuser, Basel, 2006.

[44] N. Sato, T. Aiki, Phase field equations with constraints under nonlinear dynamic boundary conditions, Commun. Appl. Anal. 5 (2001), 215-234.

[45] G. Schimperna, Abstract approach to evolution equations of phase field type and applications, J. Differential Equations 164 (2000), 395-430.

[46] L. Simon, Asymptotics for a class of nonlinear evolution equation with applications to geometric problems, Ann. of Math., 118 (1983), 525-571.

[47] R. Temam, Infinite-dimensional dynamical systems in mechanics and physics, SpringerVerlag, New York, 1997.

[48] H. Wu, S. Zheng, Convergence to equilibrium for the Cahn-Hilliard equation with dynamic boundary conditions, J. Differential Equations 204 (2004), 511-531. 
[49] H. Wu, S. Zheng, Convergence to equilibrium for the damped semilinear wave equation with critical exponent and dissipative boundary condition, Quart. Appl. Math. 64 (2006), 167-188.

[50] H. Wu, M. Grasselli, S. Zheng, Convergence to equilibrium for a parabolic-hyperbolic phase-field system with Neumann boundary conditions, Math. Models Methods Appl. Sci. 17 (2007), 125-153, 2007.

[51] H. Wu, Convergence to equilibrium for a Cahn-Hilliard model with the Wentzell boundary condition, Asympt. Anal. 54 (2007), 71-92.

[52] T.I. Zelenyak, Stabilization of solutions of boundary value problems for a second-order parabolic equation with one space variable, Differ. Urav. 4 (1968), 17-22.

[53] Z. Zhang, Asymptotic behavior of solutions to the phase-field equations with Neumann boundary conditions, Commun. Pure Appl. Anal. 4 (2005), 683-693. 